\title{
Classical Verification of Quantum Proofs
}

\author{
Zhengfeng Ji \\ Institute for Quantum Computing and School of Computer Science, \\ University of Waterloo, Waterloo, Ontario, Canada \\ State Key Laboratory of Computer Science, Institute of Software, \\ Chinese Academy of Sciences, Beijing, China
}

\begin{abstract}
We present a classical interactive protocol that verifies the validity of a quantum witness state for the local Hamiltonian problem. It follows from this protocol that approximating the non-local value of a multi-player one-round game to inverse polynomial precision is QMAhard. Our work makes an interesting connection between the theory of QMA-completeness and Hamiltonian complexity on one hand and the study of non-local games and Bell inequalities on the other.
\end{abstract}

\section{Introduction}

The concept of efficient proof verification is of fundamental importance to the theory of computation. The complexity class NP abstracts the notion of checking written proof strings by a polynomial-time deterministic verifier. It is hard to overstate the importance of NP and NPcompleteness theory $[19,33,43]$ to the development of theoretical computer science in the past several decades.

Interactive models of proof verification was proposed and studied by Babai [7] and Goldwasser, Micali, and Rackoff [26]. It is generalized to the multiple-prover setting by Ben-Or, Goldwasser, Kilian and Wigderson [10]. The efforts to understand these interactive proof systems opened the door to a series of breakthroughs in computational complexity theory (e.g., $[5,6$, $8,44,57])$.

Of particular interest to this work is the multi-player one-round game, a game theoretical model for interactive proof verification. In this model, the verifier samples a tuple of questions and sends them to the players and receives answers back from the players in one round. The players may agree on a particular strategy, but are otherwise not allowed to communicate with each other during the game. The verifier decides whether to accept or to reject based on the questions and answers. A method called oracularization [42] that enforces the functional behavior of the players provides a multi-player-game characterization of $\mathbf{N P}$, in which the questions are bit strings of length logarithmic in input length and answers are strings of constant number of bits. We emphasize that this is an exponential save in the number of bits communicated from the provers to the verifier compared with the standard way of proof communication in which the prover send the full proof string to the verifier. This is one of the reasons behind the unexpected power of multi-prover interactive proof systems and the possibility of achieving probabilistically checkable proofs $[5,6,22]$.

The study of quantum variants of proof verification systems provides both a fruitful way of understanding proof verification in the context of quantum computing and an insightful angle from which to view unique quantum phenomena such as entanglement and non-locality. 
A quantum analog of efficient verification of written proofs was proposed by Kitaev [4, 36, 37]. In this generalization, a quantum witness state plays the role of the written proof and a polynomial-time quantum computer checks whether the witness state is valid for the input. Kitaev introduces the class QMA of problems that admit efficient verifiable quantum proofs. He also establishes the quantum analog of the Cook-Levin theorem by showing that the local Hamiltonian problem, the natural quantum version of the constraint satisfaction problems, is complete for QMA. The study of local Hamiltonian problems, the structure of entanglement in the ground states of local Hamiltonians, and the quantum PCP conjecture (see e.g., [2]) form a new research direction called Hamiltonian complexity [25, 52].

Quantum interactive proof systems, a model in which a quantum polynomial-time verifier exchanges quantum messages with an all-powerful prover, were first studied by Kitaev and Watrous [38, 63]. It is now known that the class of languages expressible by such proof systems, QIP, is the same as its classical counterpart, IP [30].

In quantum multi-prover interactive proof systems, shared entanglement among the provers plays an essential role. It is known that, without shared entanglement, or with limited amount of entanglement, the collection of languages that have quantum multi-prover interactive proof systems, QMIP, equals to the classical counterpart, MIP [39] (and, hence, also equals to NEXP [8]). The classes QMIP* and MIP*, corresponding to the collections of languages that have entangled multi-prover interactive proofs with quantum and classical messages respectively, are also known to be the same [56]. There is a recent evidence showing that entangled provers may be more powerful than classical provers [23]. But a full understanding of these two complexity classes is still out of reach.

In this work, we focus on the multi-prover one-round games with entangled provers. For a multi-player one-round game, its classical value is the maximum acceptance probability that classical strategies can achieve and its non-local value is the maximum acceptance probability that entangled players can achieve. In general, the non-local value can be strictly larger than the classical value of a multi-player game. It is pointed out in [16] that this may cause problems in multi-prover interactive proof systems as provers with shared entanglement may break the soundness condition of a classically sound protocol. One striking example is given by the socalled magic square game [48,53], which has non-local value one even though it corresponds to a system of constraints with no classical solution [16]. Strong evidences are also given in that paper that the entanglement between the players may indeed weaken the power of two-player XOR games.

Several methods have been proposed to control the cheating ability of entangled provers and recover soundness in certain cases. It is proved that approximating the non-local value of a multi-player game to inverse-polynomial precision is NP-hard [28, 35]. Several natural problems arise from the study of non-locality, including the binary constraint system game [17], the quantum coloring game [14] and the game corresponding to the Kochen-Specker sets [40], are shown to be NP-hard in [31]. By proving that the multi-linearity test [8] is sound against entangled provers, Ito and Vidick proved the containment of NEXP in MIP* [29]. This was later improved to the result that three-player XOR games are NP-hard to approximate even to constant precision [62].

However, after many years of research, there is still no upper bound on the expressive power of multi-player interactive proofs with entangled provers. This leaves open the possibility that even a classical verifier may be able to design a protocol in which only provers with entanglement can follow and convince the verifier of statements that classical provers cannot prove.

In this paper, we present a multi-player one-round game for the local Hamiltonian problem in which the verifier is classical and samples questions of logarithmic size and expects answers 
of constant size. As a corollary, the problem of approximating the non-local value of a multiprover one-round game is QMA-hard, improving the NP-hardness results in previous works. It makes an interesting connection between the theory of QMA-completeness and Hamiltonian complexity on one hand, and the study of non-local games and Bell-inequalities on the other. This also provides an example that a classical verifier can design protocols making essential use of the shared entanglement between the provers and expect them to do things that is impossible for provers without shared entanglement unless $\mathbf{N P}=\mathbf{Q M A}$.

Our work can be thought of as a de-quantization of the Fitzsimons-Vidick protocol [23] of both the verifier and the messages. The verifier communicates with multiple entangled provers and delegates the quantum verification procedure to the provers. In this sense, this work is also relevant to the developments in the delegation of quantum computation and blind quantum computing [3, 13, 24, 56]. The previous works usually use a cluster state [55] or EPR states and teleportation to encode quantum computation, while our approach has the additional freedom to encode quantum data directly among the provers. This allows us to go from the delegation of quantum computation to the delegation of quantum proof verification.

The main result of this paper is stated in the following theorem.

Theorem 1. For integer $r \geq 4$, any promise problem $L=\left(L_{y e s}, L_{n o}\right)$ in $\mathbf{Q M A}$, and any instance $x$ of the problem, there exists an $r$-player one-round game and real numbers $c, s \in[0,1], c-s \geq$ $1 / \operatorname{poly}(|x|)$ such that

1. The questions are classical bit strings of length $O(\log (|x|))$.

2. The answers are classical bit strings of length $O(1)$.

3. If $x \in L_{y e s}$, then the non-local value of the game is at least c.

4. If $x \in L_{n o}$, then the non-local value of the game is at most $s$.

A direct corollary is that approximating the non-local value of a multi-player game is QMAhard.

Corollary 2. Given a multi-player one-round game in which the questions are strings of $O(\log n)$ bits and answers are of strings of $O(1)$ bits, it is QMA-hard to approximate the non-local value of the game to inverse polynomial precision.

The same problem for the classical value is obviously in NP. This means that the non-local value of multi-player one-round games is strictly harder to approximate than the classical value unless NP = QMA.

Our result has the following consequence for the multi-prover interactive proofs with entangled provers by scaling up the problem size. It is a slight improvement of the results obtained in [23]. Let $\mathbf{Q M A}_{\mathbf{E X P}}$ be the collection of problems that have quantum witnesses of exponentially many qubits verifiable by a quantum exponential-time machine, and let $\operatorname{MIP}^{*}(r, t, c, s)$ be the class of languages that have $r$-prover, $t$-round interactive proofs with a classical polynomialtime verifier, entangled provers, and completeness $c$, soundness $s$.

Corollary 3. For some choices of completeness and soundness $c, s$ and polynomial $p(n)$, with $c-$ $s=\Omega(\exp (-p(n)))$,

$$
\mathbf{Q M A}_{\mathbf{E X P}} \subseteq \mathbf{M I P}^{*}(4,1, c, s),
$$

and hence

$$
\operatorname{MIP} \subsetneq \operatorname{MIP}^{*}(4,1, c, s)
$$

unless $\mathbf{N E X P}=\mathbf{Q M A}_{\mathbf{E X P}}$. 


\subsection{Techniques and Proof Overview}

The main technical difficulty we face is how a classical verifier can check the quantum witness state distributed among a number of players. In a one-round game, the only thing that the classical verifier can collect is some information about the conditional distributions $\operatorname{Pr}(a \mid q)$ for all possible questions $q$ and answers $a$. Consider the situation of remote state certification, in which two players $A$ and $B$ share a quantum state $\rho_{A B}$ and want to convince the verifier of this fact. If the state $\rho_{A B}$ is an EPR state $(|00\rangle+|11\rangle) / \sqrt{2}$, this is possible in some sense by the verifier playing the CHSH game [15] with the players. The rigidity of the CHSH game [46, 56] implies that if the players win the CHSH game with almost optimal probability, then the state is close to the EPR state up to local isometries. If the state is mixed, however, the situation becomes problematic in a very strong sense. Suppose the two players want to prove that $\rho_{A B}$ is the Werner state [64]

$$
\rho_{\mathrm{W}}(\phi)=\frac{(d-\phi) I+(d \phi-1) \mathrm{SWAP}_{d}}{d^{3}-d},
$$

where $\operatorname{SWAP}_{d}$ is unitary operator satisfying $\operatorname{SWAP}_{d}|i, j\rangle=|j, i\rangle$ for all $0 \leq i, j \leq d-1$. It is shown by Werner [64] that, for some choices of $\phi$, the state is an entangled state, but any prescribed local measurement setting on $A$ and $B$ performed on the state $\rho_{\mathrm{W}}$ produces distributions $\operatorname{Pr}(a \mid q)$ that have local hidden variable models. That is, the distribution can be exactly reproduced by two classical players with shared randomness and no shared entangled states whatsoever!

It is natural to consider methods from the study of device-independent quantum information processing or self-testing quantum devices (e.g., [21, 45, 47, 49]). For example, such ideas have successful applications in achieving the classical command of quantum system as shown in [56]. A key ingredient behind such device independent setting is the rigidity of non-local games such as the CHSH game. By the definition of rigidity, however, the players will essentially share a specific entangled state, such as the EPR state or the GHZ state $(|000\rangle+|111\rangle) / \sqrt{2}$, and perform prescribed measurements on the state. This seems contradictory to what we need here- the ability to store the quantum witness state distributed among the players. The quantum witness state is usually an entangled state with complex structures that are far way from what EPR or GHZ states can represent.

Our solution that resolves the above mentioned difficulties is to encode the quantum witness state by certain stabilizer code and play a new game, which we call the stabilizer game, defined by the stabilizer. We prove a rigidity theorem for the stabilizer game which roughly states that the only way for the players to win the game with high probability is to share a correctly encoded state of the stabilizer code, perform measurements according to the measurement specifications given in the questions, and respond with the measurement outcome. That is, the stabilizer game we construct provides a device-independent verification of the encoding of the corresponding stabilizer code. Having both the rigidity property and the ability to encode quantum data, the stabilizer game serves as an essential tool for our work and may find other applications in deviceindependent quantum information processing. For example, in the remote state certification problem discussed above, although it is impossible for the players to certify the Werner state $\rho_{\mathrm{W}}$, the stabilizer games provide a way to certify an encoded Werner state using a stabilizer code.

In [23], quantum error correcting codes are also employed in an essential way. The intuition is that, by the quantum error correction property, there will be only one qubit of the player that can pass the encoding check of the error correcting code once the rest of the players respond with the correct qubit. In our case, the stabilizer codes have the same effect but also have an important additional use. Namely, they are responsible for enforcing the players to measure their systems according to the measurement specifications they receive. Instead of using the 
decoding circuit of the code, we measure the logical $X, Z$ operators on the encoded state. As a result, our proof directly applies to quantum error detecting codes as well.

The proof of the rigidity for the stabilizer games consists of two steps. In the first step, an idea motivated by the CHSH game is employed and we introduce the special-player stabilizer game. Using similar techniques for proving the rigidity of the $\mathrm{CHSH}$ game, we establish a pair of anti-commuting reflections in the strategy for the special player. Then, a quantity called consistency is used to promote this partial rigidity of the special-player stabilizer game to the full rigidity property of the stabilizer game itself. Consistency and another state dependent distance measure of two quantum measurements appeared before in the analysis of non-local games and are intensively used in many proofs of this paper.

We then consider the multi-qubit stabilizer game, the non-local game version of the stabilizer encoding check in [23]. We prove a partial rigidity theorem for the multi-qubit stabilizer game. This is achieved by establishing the approximate commutativity of reflections corresponding to measurement specifications on different qubits, the proof of which uses the consistency property again in an essential way. Finally, the multi-qubit stabilizer game is performed in the game for the local Hamiltonian problem with high probability to regularize the behavior of the players.

Two additional difficulties arise as the verifier only has limited access to the quantum witness state. First, since the verifier can only enforce Pauli measurements on the witness state, the measurement of energy of the local Hamiltonian uses Pauli measurements only. This will be less efficient than having the quantum data and measuring directly the POVM corresponding to each term of the Hamiltonian as is done in [23], but there will only be a constant overhead compared to the direct measurement approach.

Second, for convenience, we work with stabilizer codes whose generators are the tensor products of Pauli $I, X, Z$ operators. Alternatively, one may design an extended version of the stabilizer game using ideas from the extended CHSH game proposed in [56] to include Pauli $Y$ operators in the problem. The current form of the stabilizer game is, however, much easier to analyze. As a result, by the rigidity property, the verifier only have access to Pauli $X, Z$ measurements on the quantum witness state. This requires that the Hamiltonian in the local Hamiltonian problem has terms in the real linear span of tensor products of $I, X$ and $Z$ operators. Fortunately, as observed in [12], such restricted version of the local Hamiltonian problem remains QMA-complete.

\subsection{Open Problems}

We briefly mention several related open problems. One major weakness of our result for the multi-prover interactive proofs with entangled provers in Corollary 3 is the exponential small gap between the completeness and soundness parameters. It is an intriguing and challenging problem to improve this and show that $\mathbf{Q M A} \mathbf{E X P}_{\mathbf{E P}}$ is contained in MIP*.

Second, as we don't have any upper bound on the power of non-local games, it remains possible to prove even stronger hardness results than the QMA-hardness shown in this paper. A possible candidate problem is to show QMA(2)-hardness of non-local games.

Finally, it is an interesting question to ask whether one can improve the completeness parameter of our protocol. For example, are there non-local games with perfect completeness for $\mathbf{Q M A}_{1}$ ? It seems that, to achieve this, we need to redesign the stabilizer game and simplify the form of the local Hamiltonians to improve the efficiency of the energy measurement. 


\subsection{Organizations}

The rest of the paper is organized as follows. In Sec. 2, we introduce the notions and related concepts used in this paper. The stabilizer games are introduced and analyzed in Sec. 3. The non-local game for the local Hamiltonian problem is given and analyzed in Sec. 4.

\section{Preliminaries}

\subsection{Notions}

We use calligraphic $\mathscr{H}$ to denote Hilbert spaces, and $\mathrm{D}(\mathscr{H}), \mathrm{L}(\mathscr{H}), \operatorname{Herm}(\mathscr{H})$, $\operatorname{Pos}(\mathscr{H})$ to denote the set of density operators, bounded linear operators, Hermitian operators and positive semidefinite operators on $\mathscr{H}$. Two-dimensional Hilbert spaces $\mathbb{C}^{2}$ corresponding to a qubit is denoted by $\mathscr{B}$. For two Hermitian operators $M, N \in \operatorname{Herm}(\mathscr{H})$, we write $M \leq N$ to mean $N-M \in \operatorname{Pos}(\mathscr{H})$. For matrix $M,|M|$ is defined to be $\sqrt{M^{\dagger} M}$. For a string $x,|x|$ denotes its length. For a positive integer $k,[k]$ is the abbreviation of the set $\{1,2, \ldots, k\}$. For two complex numbers $x, y$, we use $x \approx_{\epsilon} y$ as a shorthand notion for $|x-y| \leq O(\epsilon)$. Two-qubit unitary gates CNOT and SWAP are defined as

$$
\mathrm{CNOT}|i, j\rangle=|i\rangle|i \oplus j\rangle, \quad \operatorname{SWAP}|i, j\rangle=|j, i\rangle,
$$

for $i, j \in\{0,1\}$.

Let $\rho \in \mathrm{D}(\mathscr{H})$ be a quantum state on $\mathscr{H}$. For operators $M, N \in \mathrm{L}(\mathscr{H})$, introduce the following notions

$$
\begin{aligned}
\operatorname{tr}_{\rho}(M) & =\operatorname{tr}(M \rho), \\
\langle M, N\rangle_{\rho} & =\operatorname{tr}_{\rho}\left(M^{\dagger} N\right), \\
\|M\|_{\rho} & =\sqrt{\langle M, M\rangle_{\rho}} .
\end{aligned}
$$

It is straightforward to verify that $\langle\cdot, \cdot\rangle_{\rho}$ is a semi-inner-product, $\|\cdot\|_{\rho}$ is a seminorm and they become an inner product and a norm, respectively, when $\rho$ is a full-rank state. In particular, the Cauchy-Schwarz inequality holds

$$
\left|\langle M, N\rangle_{\rho}\right| \leq\|M\|_{\rho}\|N\|_{\rho},
$$

or more explicitly,

$$
\left|\operatorname{tr}_{\rho}\left(M^{\dagger} N\right)\right| \leq\left[\operatorname{tr}_{\rho}\left(M^{\dagger} M\right) \operatorname{tr}_{\rho}\left(N^{\dagger} N\right)\right]^{1 / 2} .
$$

For state $\rho \in \mathrm{D}\left(\mathscr{H}_{A} \otimes \mathscr{H}_{B}\right)$, and an operator $M \in \mathrm{L}\left(\mathscr{H}_{A}\right)$, we may also write $\operatorname{tr}_{\rho}(M)$ even though the state $\rho$ and the operator $M$ do not act on the same space. In this case, it is understood that $\operatorname{tr}_{\rho}(M)=\operatorname{tr}_{\rho_{A}}(M)$ where $\rho_{A}$ is the reduced state of $\rho$ on system $A$. This is one reason that makes $\operatorname{tr}_{\rho}(\cdot)$ easy to use as it is not necessary to specify the correct reduced state explicitly all the time.

A quantum measurement is described by a collection $M=\left\{M^{a}\right\}$ of measurement operators. These are operators acting on the state space $\mathscr{H}$ of the system being measured. In this paper, we always use superscript to index the measurement outcomes and subscript to index different quantum measurements. The measurement operators satisfy the completeness equation

$$
\sum_{a}\left(M^{a}\right)^{\dagger} M^{a}=I .
$$


If the state of the system before the measurement is $\rho \in \mathrm{D}(\mathscr{H})$, the probability that outcome $a$ occurs is given by

$$
\operatorname{tr}\left(\left(M^{a}\right)^{\dagger} M^{a} \rho\right)=\left\|M^{a}\right\|_{\rho}^{2},
$$

and the post-measurement state of the system is

$$
\frac{M^{a} \rho\left(M^{a}\right)^{\dagger}}{\left\|M^{a}\right\|_{\rho}^{2}} .
$$

A positive operator valued measure (POVM) is a collection of positive semidefinite operators $\left\{M^{a}\right\}$, such that

$$
\sum_{a} M^{a}=I
$$

POVMs give descriptions of quantum measurements when the post-measurement state is not important in the analysis. The probability that the measurement has outcome $a$ on state $\rho$ is given by $\operatorname{tr}_{\rho}\left(M^{a}\right)$.

A projective quantum measurement is described by $\left\{M^{a}\right\}$ where each operator $M^{a}$ is a projection and $\sum_{a} M^{a}=I$. A reflection $R$ is a Hermitian matrix squared to $I$. It naturally describes a two-outcome projective quantum measurement $\left\{R^{a}\right\}$ where

$$
R^{a}=\frac{I+(-1)^{a} R}{2}
$$

for $a=0,1$. The Pauli operators

$$
I=\left[\begin{array}{ll}
1 & 0 \\
0 & 1
\end{array}\right], \quad X=\left[\begin{array}{ll}
0 & 1 \\
1 & 0
\end{array}\right], \quad Y=\left[\begin{array}{rr}
0 & -i \\
i & 0
\end{array}\right], \quad Z=\left[\begin{array}{rr}
1 & 0 \\
0 & -1
\end{array}\right],
$$

are examples of 2-by-2 reflections. We also use $\sigma_{0}, \sigma_{1}, \sigma_{2}, \sigma_{3}$ to represent the four Pauli operators. A multi-qubit Pauli operator is of $X Z$-form if each tensor factor is one of $I, X$ and $Z$. A Hermitian matrix $H$ is of $X Z$-form if it is in the real linear span of $X Z$-from Pauli operators.

Define $X^{\prime}$ and $Z^{\prime}$ as

$$
X^{\prime}=\frac{X+Z}{\sqrt{2}}, \quad Z^{\prime}=\frac{X-Z}{\sqrt{2}},
$$

and $W$ as

$$
W=\cos (\pi / 8) X+\sin (\pi / 8) Z .
$$

It is easy to verify that $W$ is a reflection and

$$
\begin{aligned}
X & =W X^{\prime} W, & X^{\prime} & =W X W, \\
Z & =W Z^{\prime} W, & Z^{\prime} & =W Z W .
\end{aligned}
$$

That is, under the conjugation of $W, X^{\prime}$ and $Z^{\prime}$ are mapped to $X$ and $Z$ respectively, and vice versa. The reflections $X, Z, X^{\prime}, Z^{\prime}$ and $W$ are illustrate in Fig. 1. They play an important role in the CHSH game and the stabilizer games introduced in this paper.

\subsection{Non-Local Games}

A multi-player one-round game involves two or more players and a verifier who communicates with the players classically in one round. The verifier samples questions and sends them out to the players and expects to receive answers back. He then decides whether to accept or reject 


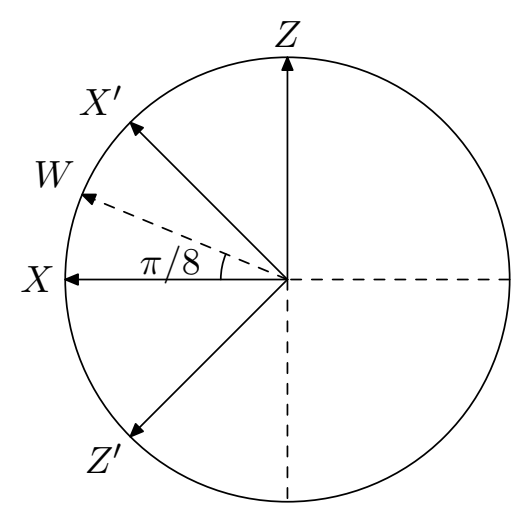

Figure 1: Reflections $X, Z, X^{\prime}, Z^{\prime}, W$ in the $x, z$-plane of the Bloch sphere.

based on the questions and answers. The players are allowed to agree on a strategy before the game starts, but cannot communicate with each other during the game.

Let there be $r$ players, (1), (2), .., (r). Let $\Gamma^{(i)}$ be a finite set of questions for player $(i)$ and $\Lambda^{(i)}$ be a finite set of possible answers from player $(i)$. An $r$-player game is defined by a distribution $\pi$ over $\prod_{i=1}^{r} \Gamma^{(i)}$ and a function $V: \prod_{i=1}^{r} \Lambda^{(i)} \times \prod_{i=1}^{r} \Gamma^{(i)} \rightarrow[0,1]$, specifying the acceptance probability. By a convexity argument, it suffices to consider the strategy of classical players described by functions $f^{(i)}: \Gamma^{(i)} \rightarrow \Lambda^{(i)}$. The value of the strategy is the acceptance probability

$$
\omega=\underset{q \sim \pi}{\mathbb{E}} V(a(q), q),
$$

for $q=\left(q_{1}, q_{2}, \ldots, q_{r}\right)$ distributed according to $\pi$ and $a(q)=\left(f^{(1)}\left(q_{1}\right), f^{(2)}\left(q_{2}\right), \ldots, f^{(r)}\left(q_{r}\right)\right)$. The classical value of the game is the maximum of the values of all classical strategies. An XOR game is a multi-player game in which each player answers a bit $a_{i}$ and the verifier accepts or rejects depending only on the parity of $\bigoplus_{i=1}^{r} a_{i}$. More precisely, there is a function $\hat{V}:\{0,1\} \times \prod_{i=1}^{r} \Gamma^{(i)} \rightarrow$ $[0,1]$ such that

$$
V(a, q)=\hat{V}\left(\bigoplus_{i=1}^{r} a_{i}, q\right)
$$

for all $q \in \prod_{i=1}^{r} \Gamma^{(i)}, a \in \prod_{i=1}^{r} \Lambda^{(i)}$. In most of the games considered in this paper, the verifier accepts or rejects only depending on the parity of some, not all, of the answer bits. We call them generalized XOR games.

In a non-local game, the players are allowed to share an arbitrary entangled state before the game starts. A quantum strategy $\mathscr{S}$ for the non-local game is described by the shared state $\rho$, the measurements $\left\{M_{q_{i}}^{(i)}\right\}$ that player $(i)$ performs when the question is $q_{i} \in \Gamma^{(i)}$. The value of the strategy is defined as

$$
\omega^{*}(\mathscr{S})=\underset{q \sim \pi}{\mathbb{E}} \sum_{a}\left[\operatorname{tr}_{\rho}\left(\bigotimes_{i=1}^{r} M_{q_{i}}^{(i), a_{i}}\right) V(a, q)\right],
$$

for $a=\left(a_{1}, a_{2}, \ldots, a_{r}\right)$ and $q=\left(q_{1}, q_{2}, \ldots, q_{r}\right)$. The non-local value of the game is the supremum of the values of all quantum strategies.

The CHSH game [15] is arguably one of the most important non-local games. It arises from the study of fundamental questions in quantum mechanics such as entanglement and nonlocality via Bell inequalities [9]. The CHSH game is a two-player XOR game. The verifier samples two bits $q_{1}$ and $q_{2}$ independently and uniformly at random, sends $q_{1}$ to the first player and $q_{2}$ to the second player. The verifier accepts if and only if two answer bits $a_{1}$ and $a_{2}$ satisfy 
$a_{1} \oplus a_{2}=q_{1} \wedge q_{2}$. The classical value of the game is $3 / 4=0.75$ and the non-local value of the game is $\omega_{\mathrm{CHSH}}^{*}=(2+\sqrt{2}) / 4 \approx 0.85[59]$.

In an optimal strategy for the CHSH game, the players share an EPR state $(|00\rangle+|11\rangle) / \sqrt{2}$, and the first player obtains the answer by measuring $X$ (or $Z$ ) if the question is 0 (or 1 respectively), while the second player measures $X^{\prime}$ (or $Z^{\prime}$ ) in Eq. (2) if the question is 0 (or 1). The rigidity property of the $\mathrm{CHSH}$ game roughly states that this is essentially the only strategy for the players to achieve the non-local value, up to local isometries. Furthermore, any strategy that has value close to the game value must be close to this optimal strategy in some sense. Rigidity of the CHSH game and other non-local games has found interesting applications in certifiable randomness generation (e.g., [18, 50, 54, 60]), device-independent quantum cryptography (e.g., [1, 50, 61]) and classical command of quantum systems [56].

\subsection{Quantum Proofs and Local Hamiltonian Problems}

The idea of efficient proof verification of NP has a natural quantum generalization given in the following definition.

Definition 4. The complexity class QMA is the set of promise problems $L=\left(L_{y e s}, L_{n o}\right)$ such that there is a polynomial $p(\cdot)$ and a quantum polynomial-time verifier $V$, and

- Completeness. If $x \in L_{y e s}$, there exists a state $|\psi\rangle$ of $p(|x|)$ qubits,

$$
\operatorname{Pr}[V \text { accepts }|x\rangle \otimes|\psi\rangle] \geq \frac{2}{3},
$$

- Soundness. If $x \in L_{n o}$, then for all state $|\psi\rangle$ of $p(|x|)$ qubits,

$$
\operatorname{Pr}[V \text { accepts }|x\rangle \otimes|\psi\rangle] \leq \frac{1}{3} .
$$

Definition 5 (Local Hamiltonian Problems). An instance of the $k$-local Hamiltonian problem of $n$-qubits is described by the tuple $(H, a, b)$, where the Hamiltonian $H=\sum_{j=1}^{m} H_{j}$ and each term

$H_{j}$ acts non-trivially on at most $k$-qubits, $H_{j}$ is positive semidefinite and $\left\|H_{j}\right\| \leq 1, a, b \in \mathbb{R}$ are numbers satisfying $b-a \geq 1 / \operatorname{poly}(n)$. Let the ground state energy of the Hamiltonian $H$ be $\lambda_{\min }=$ $\min _{\rho \in \mathrm{D}}\langle H, \rho\rangle$. In the $k$-local Hamiltonian problem, $(H, a, b)$ is a yes-instance if $\lambda_{\min } \leq$ am and $a$ no-instance if $\lambda_{\min } \geq b m$.

The quantum analog of the Cook-Levin theorem by Kitaev states that the $k$-local Hamiltonian problem is QMA-complete for $k \geq 5$ [4, 36]. This was later improved to 2-local and more physical Hamiltonians in a series of works (see e.g., [20, 34, 51]).

\subsection{Quantum Error Correction and Stabilizer Formalism}

A quantum error correcting code encodes a number of qubits, called the logical qubits, into a larger number of physical qubits with the aim of protecting the quantum information in the logical qubits from certain types of noises.

The stabilizer formalism provides a convenient language and great amount of examples of quantum error correcting codes. We present several relevant definitions and facts about the stabilizer codes and refer the reader to the thesis of Gottesman [27] for more details. Let $\mathscr{P}_{r}$ be the group generated by the $r$-fold tensor product of Pauli operators

$$
\mathscr{P}_{r}=\left\{e^{i \phi} \bigotimes_{j=1}^{r} P_{j}, \text { for } \phi \in\{0, \pi / 2, \pi, 3 \pi / 2\}, P_{j} \in\{I, X, Y, Z\}\right\} .
$$


A stabilizer $\mathfrak{S}$ is an abelian subgroup of $\mathscr{P}_{r}$ not containing $-I^{\otimes r}$. The stabilizer provides a succinct description of a subspace of $\left(\mathbb{C}^{2}\right)^{\otimes r}$, the simultaneous +1-eigenspace of the operators in $\mathfrak{S}$. This subspace is called the code space of the stabilizer. A set of operators in $\mathfrak{S}$ is called the generators of $\mathfrak{S}$ if they generate the group $\mathfrak{S}$.

The weight of an operator in $\mathscr{P}_{r}$ is the number of non-identity tensor factors in it. Let $C(\mathfrak{S})$ be the centralizer of $\mathfrak{S}$ in $\mathscr{P}_{r}$, the set of operators in $\mathscr{P}_{r}$ that commutes with $\mathfrak{S}$. The distance of the stabilizer code is $d$ if there is no operator of weight less than $d$ in $C(\mathfrak{S})-\mathfrak{S}$. The logical $X$ and $Z$ operators $L_{X}$ and $L_{Z}$ are a pair of anti-commuting operators in $C(\mathfrak{S})-\mathfrak{S}$.

As a simple example, the operators $X X$ and $Z Z$ generate a stabilizer for the EPR state. The famous five-qubit code $[11,41]$ has a stabilizer representation given in Fig. 2a. The five-qubit code encodes one logical qubit in five physical qubits and has distance 3 . The logical $X$ and $Z$ operators are $X^{\otimes 5}$ and $Z^{\otimes 5}$. This will be the stabilizer code we use most of the time as the example. Operators $X^{\otimes 4}$ and $Z^{\otimes 4}$ generate the stabilizer for the four-qubit quantum error detecting code. It has distance 2 and encodes two qubits. The operators $X X I I$ and $Z I Z I$ form a pair of anti-commuting operators and can serve as the logical $X$ and $Z$ operators. There is another pair of the logical operators for the other encoded qubit that we do not use.

\subsection{State-Dependent Distance Measures of Quantum Measurements}

We introduce a distance measure and a consistency measure of quantum measurements that will be helpful in our treatment of non-local games. They grow out of the study of non-local games [28, 29, 62] and may be useful in more general contexts. A common feature of them is that they are both state-dependent.

A general question that one usually faces is what the consequences are if measurement $\left\{M_{1}^{a}\right\}$ is used in place of $\left\{M_{0}^{a}\right\}$ in a non-local game by one of the players. Will it change the overall analysis at lot? More concretely, suppose that the state of a joint quantum system $\mathscr{H}_{A}$ and $\mathscr{H}_{B}$ is $\rho$ and that $\left\{M_{0}^{a}\right\},\left\{M_{1}^{a}\right\}$ are quantum measurements on system $A$. The post-measurement states are

$$
\rho_{i}=\sum_{a}|a\rangle\langle a| \otimes M_{i}^{a} \rho\left(M_{i}^{a}\right)^{\dagger},
$$

for $i=0,1$, respectively, depending on which measurement is performed. By the monotonicity of the trace distance, the difference will be bounded by $D_{\operatorname{tr}}\left(\rho_{0}, \rho_{1}\right)$ no matter what operation follows the measurement. In particular, in a non-local game, if Bob measures on his system $\mathscr{H}_{B}$ and then the verifier makes the decision, the acceptance probabilities will differ by at most $D_{\operatorname{tr}}\left(\rho_{0}, \rho_{1}\right)$.

The quantity defined next provides a way to bound the distance $D_{\operatorname{tr}}\left(\rho_{0}, \rho_{1}\right)$.

Definition 6. For two quantum measurements $M_{i}=\left\{M_{i}^{a}\right\}$ with $i=0,1$ that have the same set of possible outcomes, define

$$
d_{\rho}\left(M_{0}, M_{1}\right)=\left[\sum_{a}\left\|M_{0}^{a}-M_{1}^{a}\right\|_{\rho}^{2}\right]^{1 / 2} \text {. }
$$

More explicitly,

$$
d_{\rho}\left(M_{0}, M_{1}\right)=\left[2-2 \operatorname{Re} \sum_{a} \operatorname{tr}_{\rho}\left(\left(M_{0}^{a}\right)^{\dagger} M_{1}^{a}\right)\right]^{1 / 2} .
$$

Lemma 7. Let $M_{i}=\left\{M_{i}^{a}\right\}$ for $i=0,1$ be two quantum measurements with the same set of possible outcomes, and $\rho_{i}$ be the post-measurement states in Eq. (4). Then

$$
D_{\operatorname{tr}}\left(\rho_{0}, \rho_{1}\right) \leq d_{\rho}\left(M_{0}, M_{1}\right) .
$$


Proof. Let $|\psi\rangle \in \mathscr{H}_{A} \otimes \mathscr{H}_{B} \otimes \mathscr{H}_{C}$ be a purification of $\rho$. Then

$$
\begin{aligned}
D_{\operatorname{tr}}\left(\rho_{0}, \rho_{1}\right) & \leq D_{\operatorname{tr}}\left(\sum_{a}|a\rangle \otimes\left(M_{0}^{a}|\psi\rangle\right), \sum_{a}|a\rangle \otimes\left(M_{1}^{a}|\psi\rangle\right)\right) \\
& =\left[1-\left|\sum_{a}\left\langle\psi\left|\left(M_{0}^{a}\right)^{\dagger} M_{1}^{a}\right| \psi\right\rangle\right|^{2}\right]^{1 / 2} \\
& \leq\left[2\left(1-\left|\sum_{a}\left\langle\psi\left|\left(M_{0}^{a}\right)^{\dagger} M_{1}^{a}\right| \psi\right\rangle\right|\right)\right]^{1 / 2} \\
& \leq\left[2-2 \operatorname{Re} \sum_{a} \operatorname{tr}_{\rho}\left(\left(M_{0}^{a}\right)^{\dagger} M_{1}^{a}\right)\right]^{1 / 2} \\
& =d_{\rho}\left(M_{0}, M_{1}\right) .
\end{aligned}
$$

The first inequality follows from the monotonicity of the trace distance. The second line follows by a direct calculation of the trace distance for two pure states. The third line is from $1-x^{2} \leq$ $2(1-x)$ for $x \in[0,1]$.

As discussed above, a direct corollary of the above lemma is that when measurement $M_{0}$ is replaced with $M_{1}$ in a strategy for a non-local game using shared state $\rho$, the acceptance probability change by at most $d_{\rho}\left(M_{0}, M_{1}\right)$. This claim works for all types of quantum measurements including the general quantum measurement, POVMs, projective quantum measurements and binary projective measurements described by reflections.

For $M_{i}=\left\{M_{i}^{a}\right\}, i=0,1$, describing two POVMs that satisfy $\sum_{a} M_{i}^{a}=I$, define the corresponding distance as

$$
d_{\rho}\left(M_{0}, M_{1}\right)=\left[2-2 \operatorname{Re} \sum_{a} \operatorname{tr}_{\rho}\left(\sqrt{M_{0}^{a}} \sqrt{M_{1}^{a}}\right)\right]^{1 / 2} .
$$

For projective measurements $M_{i}=\left\{M_{i}^{a}\right\}$, define

$$
d_{\rho}\left(M_{0}, M_{1}\right)=\left[2-2 \operatorname{Re} \sum_{a} \operatorname{tr}_{\rho}\left(M_{0}^{a} M_{1}^{a}\right)\right]^{1 / 2} .
$$

Finally, for reflections $R_{0}, R_{1}$, let

$$
R_{i}^{a}=\frac{I+(-1)^{a} R_{i}}{2}
$$

be the projective measurement operators correspond to $R_{i}$. Define

$$
\begin{aligned}
d_{\rho}\left(R_{0}, R_{1}\right) & =d_{\rho}\left(\left\{R_{0}^{a}\right\},\left\{R_{1}^{a_{1}}\right\}\right) \\
& =\left[2-2 \operatorname{Re} \sum_{a} \operatorname{tr}_{\rho}\left(\frac{I+(-1)^{a} R_{0}}{2} \frac{I+(-1)^{a} R_{1}}{2}\right)\right]^{1 / 2} \\
& =\left[1-\operatorname{Retr}_{\rho}\left(R_{0} R_{1}\right)\right]^{1 / 2} .
\end{aligned}
$$

It is easy to verify that $d_{\rho}$ satisfy the triangle inequality.

Lemma 8. Let $M_{0}, M_{1}, M_{2}$ be three measurements on state $\rho$. Then

$$
d_{\rho}\left(M_{0}, M_{2}\right) \leq d_{\rho}\left(M_{0}, M_{1}\right)+d_{\rho}\left(M_{1}, M_{2}\right) .
$$

The next important quantity measures the consistency of two quantum measurements. 
Definition 9. Let $\rho \in \mathrm{D}\left(\mathscr{H}_{A} \otimes \mathscr{H}_{B}\right)$ be the shared state between system $A$ and $B$, let $M=\left\{M^{a}\right\}$, $N=\left\{N^{a}\right\}$ be POVMs on system A and B respectively having the same set of possible outcomes. Define the consistency of $M, N$ on state $\rho$ as

$$
C_{\rho}(M, N)=\sum_{a} \operatorname{tr}_{\rho}\left(M^{a} \otimes N^{a}\right) .
$$

$M$ and $N$ are called $\epsilon$-consistent on state $\rho$ if $C_{\rho}(M, N) \geq 1-\epsilon$.

For two reflections $R, S$, let $\left\{R^{a}\right\},\left\{S^{a}\right\}$ be their corresponding projective measurements. Define

$$
C_{\rho}(R, S)=C_{\rho}\left(\left\{R^{a}\right\},\left\{S^{a_{1}}\right\}\right)=\frac{1+\operatorname{tr}_{\rho}(R \otimes S)}{2} .
$$

The condition $\operatorname{tr}_{\rho}(R \otimes S) \approx_{\epsilon} 1$, or equivalently, $R, S$ are $O(\epsilon)$-consistent on $\rho$, can be thought of as a quantitative way of saying that $\rho$ is approximately stabilized by $R \otimes S$.

The consistency of measurements puts strong structural constraints on the strategies of nonlocal game. It will be a key ingredient in our proof of the main result. In the following lemma, it is proved that if two measurements $M_{0}, M_{1}$ are consistent with the same measurement $N$, then $M_{0}, M_{1}$ must be close to each other in terms of the distance $d_{\rho}$.

Lemma 10. Let $\rho \in \mathrm{D}\left(\mathscr{H}_{A} \otimes \mathscr{H}_{B}\right)$ be a state on system $A$ and $B$. Let $M_{i}=\left\{M_{i}^{a}\right\}$ for $i=0,1$ be POVMs on system $A, N=\left\{N^{a}\right\}$ be a POVM on system $B$. If

$$
C_{\rho}\left(M_{i}, N\right) \geq 1-\epsilon,
$$

for $i=0,1$, then

$$
d_{\rho}\left(M_{0}, M_{1}\right) \leq O(\sqrt{\epsilon})
$$

Proof. First prove that

$$
\sum_{a} \operatorname{tr}_{\rho}\left[\left(1-\sqrt{M_{0}^{a}}\right)\left(1-\sqrt{M_{1}^{a}}\right) \otimes N^{a}\right] \approx_{\epsilon} 0 .
$$

By the Cauchy-Schwarz inequality, the absolute value of the left hand side is at most

$$
\begin{aligned}
& \sum_{a} \sqrt{\operatorname{tr}_{\rho}\left[\left(1-\sqrt{M_{0}^{a}}\right)^{2} \otimes N^{a}\right] \operatorname{tr}_{\rho}\left[\left(1-\sqrt{M_{1}^{a}}\right)^{2} \otimes N^{a}\right]} \\
\leq & \sum_{a} \sqrt{\operatorname{tr}_{\rho}\left[\left(1-M_{0}^{a}\right) \otimes N^{a}\right] \operatorname{tr}_{\rho}\left[\left(1-M_{1}^{a}\right) \otimes N^{a}\right]} \\
\leq & \sqrt{\sum_{a} \operatorname{tr}_{\rho}\left[\left(1-M_{0}^{a}\right) \otimes N^{a}\right] \sum_{a} \operatorname{tr}_{\rho}\left[\left(1-M_{1}^{a}\right) \otimes N^{a}\right]} \\
\leq & \epsilon
\end{aligned}
$$

where the first inequality follows from the fact that $(1-\sqrt{x})^{2} \leq 1-x$ for $x \in[0,1]$, the second inequality is another Cauchy-Schwarz inequality and the last inequality follows from the condition that $C_{\rho}\left(M_{i}, N\right) \geq 1-\epsilon$.

Similarly, by using the Cauchy-Schwarz inequality twice, we have

$$
\sum_{a} \operatorname{tr}_{\rho}\left[\sqrt{M_{0}^{a}} \sqrt{M_{1}^{a}} \otimes\left(1-N^{a}\right)\right] \approx_{\epsilon} 0
$$

Adding Eqs. (9) and (10) gives

$$
\begin{aligned}
\sum_{a} \operatorname{tr}_{\rho}\left[\sqrt{M_{0}^{a}} \sqrt{M_{1}^{a}}\right] & \approx_{\epsilon} \sum_{a} \operatorname{tr}_{\rho}\left[\sqrt{M_{0}^{a}} \otimes N^{a}\right]+\sum_{a} \operatorname{tr}_{\rho}\left[\sqrt{M_{1}^{a}} \otimes N^{a}\right]-1 \\
& \approx_{\epsilon} 1+1-1=1 .
\end{aligned}
$$

The lemma follows by the definition of $d_{\rho}$ for POVMs. 
In the analysis, it is useful to have a quantity characterizing the approximate commutativity of two projective measurements $M=\left\{M^{a}\right\}$ and $N=\left\{N^{a}\right\}$ on a state $\rho$. The quantity we choose is

$$
\sum_{a}\left\|\left[M^{a}, N^{a}\right]\right\|_{\rho}^{2} .
$$

For reflections $R, S$, let $\left\{R^{a}\right\}$ and $\left\{S^{a}\right\}$ be the projective measurements correspond to $R$ and $S$ respectively. The commutativity of these two measurements on state $\rho$

$$
\begin{aligned}
\sum_{a \in\{0,1\}}\left\|\left[R^{a}, S^{a}\right]\right\|_{\rho}^{2} & =\sum_{a \in\{0,1\}}\left\|\left[\frac{I+(-1)^{a} R}{2}, \frac{I+(-1)^{a} S}{2}\right]\right\|_{\rho}^{2} \\
& =\frac{1}{8}\|[R, S]\|_{\rho}^{2} .
\end{aligned}
$$

For this reason, $\|[R, S]\|_{\rho}^{2}$ equivalently serves as a bound on the approximate commutativity of the two projective measurements defined by $R, S$.

\section{Stabilizer Games and Rigidity}

\subsection{CHSH Game Revisited}

Before introducing the stabilizer games, it is beneficial to revisit the CHSH game in the stabilizer formalism.

Recall that the EPR state $|\Phi\rangle=(|00\rangle+|11\rangle) / \sqrt{2}$ is a stabilizer state defined by two generators $g_{1}=X X$ and $g_{2}=Z Z$, and the eigenstate of eigenvalue 2 of the operator $g_{1}+g_{2}$. If a verifier has trusted measuring devices, it suffices to perform the projective measurements associated with the reflections $g_{1}, g_{2}$ to check whether the state is an EPR state. However, this simple measurement setting does not correspond to any non-trivial schemes that allow device-independent certification of the EPR state.

In the CHSH game, one of the two players is asked to measure her share of $|\Phi\rangle$ with $X, Z$, the other is asked to measure $X^{\prime}, Z^{\prime}$ in Eq. (2), the $\pi / 4$ rotated versions of $X, Z$. This motivates us to consider the generators $g_{1}^{\prime}=X X^{\prime}$ and $g_{2}^{\prime}=Z Z^{\prime}$. By the conjugation relation of $X, Z$ and $X^{\prime}, Z^{\prime}$, they generate a stabilizer for the state $\left|\Phi^{\prime}\right\rangle=I \otimes W|\Phi\rangle$ and

$$
\left\langle\Phi^{\prime}\left|\sum_{i=1}^{2} g_{i}^{\prime}\right| \Phi^{\prime}\right\rangle=2
$$

Expanding the $X^{\prime}, Z^{\prime}$ in $g_{1}^{\prime}$ and $g_{2}^{\prime}$ using $X$ and $Z$ gives four operators

$$
h_{1}=X X, \quad h_{2}=X Z, \quad h_{3}=Z X, \quad h_{4}=-Z Z,
$$

such that $h_{1}+h_{2}=\sqrt{2} g_{1}^{\prime}$ and $h_{3}+h_{4}=\sqrt{2} g_{2}^{\prime}$. The four operators $h_{i}$ in Eq. (12) recover exactly the CHSH game by encoding the Pauli operators $X, Z$ in $h_{i}$ as questions 0,1 and the sign \pm 1 of $h_{i}$ as the expected parity of answers. The Eq. (11) becomes

$$
\left\langle\Phi^{\prime}\left|\sum_{i=1}^{4} h_{i}\right| \Phi^{\prime}\right\rangle=2 \sqrt{2}
$$

which is an explanation of the $\sqrt{2}$ quantum advantage in the CHSH game. 


\subsection{Special-Player Stabilizer Game}

In this section, we introduce the stabilizer games with a special player. The construction works with any non-trivial stabilizer code that has a set of generators all in the tensor product form of $I, X, Z$.

Consider the generators of the stabilizer group for the five-qubit quantum code in Fig. 2a. Its code space is the two-dimensional eigenspace of eigenvalue 4 of the operator $\sum_{j=1}^{4} g_{j}$, where $g_{j}$ 's are operators in Fig. 2a.

\begin{tabular}{c|c}
\hline \hline Name & Operator \\
\hline$g_{1}$ & $I X Z Z X$ \\
$g_{2}$ & $X I X Z Z$ \\
$g_{3}$ & $Z X I X Z$ \\
$g_{4}$ & $Z Z X I X$ \\
\hline \hline
\end{tabular}

(a) Standard generators

\begin{tabular}{c|c}
\hline \hline Name & Operator \\
\hline$g_{1}^{\prime}$ & $I X Z Z X^{\prime}$ \\
$g_{2}^{\prime}$ & $X I X Z Z^{\prime}$ \\
$g_{3}^{\prime}$ & $Z X I X Z^{\prime}$ \\
$g_{4}^{\prime}$ & $Z Z X I X^{\prime}$ \\
\hline \hline
\end{tabular}

(b) Generators with the last qubit rotated.

Figure 2: Stabilizer generators for the five-qubit code.

Motivated by the CHSH game, we apply the $\pi / 4$-trick to the last column of the four generators in Fig. 2a. That is, we replace $X$ and $Z$ in the last column with $X^{\prime}$ and $Z^{\prime}$ in Eq. (2). This gives us another set of generators, as in Fig. $2 b$, which generates the stabilizer for the five-qubit code with the last qubit rotated by the single-qubit unitary $W$ in Eq. (3). If $|\psi\rangle$ is in the code space of the five-qubit code, state

$$
\left|\psi^{\prime}\right\rangle=(I \otimes W)|\psi\rangle
$$

is in the eigenspace of $\sum_{j=1}^{4} g_{j}^{\prime}$ with eigenvalue 4 .

Expanding the primed $X, Z$ operators in each of the generators in Fig. 2b into $X, Z$, we get a set of eight operators $h_{j}$ as in Fig. 3a. For state $\left|\psi^{\prime}\right\rangle$ in Eq. (13),

$$
\left\langle\psi^{\prime}\left|\sum_{j=1}^{8} h_{j}\right| \psi^{\prime}\right\rangle=4 \sqrt{2} .
$$

The table in Fig. 3b is obtained by translating the operators $I, X, Z$ in Fig. $3 \mathrm{a}$ to the questions in the alphabet of $*, 0,1,2,3$. The $I$ operator is always translated to $*$, which denotes a null question. The verifier will not ask anything to the player and do not expect any answers if the question is the null question $*$. For convenience, we sometimes assume that the verifier will replace $*$ with either 0 or 1 as the question and ignore the answers corresponding to this question. The operators $X, Z$ are translated to 0,1 respectively in the first four columns and to 2, 3 respectively in the last column. One can of course also use 0,1 in the last the column and the change is only for later convenience. Finally, the parity column is read off from the \pm 1 signs in the $h_{i}$ operators.

The table in Fig. 3b specifies a five-player game in Fig. 4 called the Special-Player Stabilizer Game. In the game, the verifier randomly selects four players each time, asks a question encoded with a single bit and expects a single bit answer. He accepts or rejects depending on the parity of the answers received. The fifth player is special because of the $\pi / 4$-rotation applied on the fifth column of the stabilizer generators. This breaks the translation invariance of the five-qubit code and, in the honest strategy, the fifth player performs differently from other players.

It turns out that the special-player stabilizer game in Fig. 4 has non-local value

$$
\omega_{\mathrm{SPS}}^{*}=\frac{2+\sqrt{2}}{4},
$$




\begin{tabular}{c|r}
\hline \hline Name & \multicolumn{1}{|c}{ Operator } \\
\hline$h_{1}$ & $I X Z Z X$ \\
$h_{2}$ & $I X Z Z Z$ \\
\hline$h_{3}$ & $X I X Z X$ \\
$h_{4}$ & $-X I X Z Z$ \\
\hline$h_{5}$ & $Z X I X X$ \\
$h_{6}$ & $-Z X I X Z$ \\
\hline$h_{7}$ & $Z Z X I X$ \\
$h_{8}$ & $Z Z X I Z$ \\
\hline \hline
\end{tabular}

(a) Eight operators obtained from Fig. $2 b$.

\begin{tabular}{c|cccccc}
\hline \hline Parity & \multicolumn{4}{|c}{ Question } \\
\hline 0 & $*$ & 0 & 1 & 1 & 2 \\
\hline 0 & $*$ & 0 & 1 & 1 & 3 \\
\hline 0 & 0 & $*$ & 0 & 1 & 2 \\
\hline 1 & 0 & $*$ & 0 & 1 & 3 \\
\hline 0 & 1 & 0 & $*$ & 0 & 2 \\
\hline 1 & 1 & 0 & $*$ & 0 & 3 \\
\hline 0 & 1 & 1 & 0 & $*$ & 2 \\
\hline 0 & 1 & 1 & 0 & $*$ & 3 \\
\hline \hline
\end{tabular}

(b) The table for the game with a special player.

Figure 3: Translation from measurement operators to game specifications.

\section{Special-Player Stabilizer Game}

Let the $s_{j}$ for $j \in[8]$ be the eight entries in the parity column of Fig. $3 \mathrm{~b}$ and let $w_{j}=$ $\left(w_{j, i}\right)$ be the $j$-th row of the question column. The verifier does the following:

1. Select an index $j \in[8]$ uniformly at random.

2. For $i \in[5]$, send $w_{j, i}$ in Fig. $3 \mathrm{~b}$ to the player $(i)$ if $w_{j, i}$ is not $*$, the null question.

3. Receive a bit $a^{(i)}$ from player $(i)$ if she was asked a question.

4. Accept if and only if the parity of the answers $\bigoplus_{i} a^{(i)}$ equals $s_{j}$.

Figure 4: Special-player stabilizer game for the five-qubit code. The fifth player is the special player.

the same as that of the CHSH game. The following strategy achieves the value. The five players share the state $\left|\psi^{\prime}\right\rangle$ as in Eq. (13) and measure $X$ (or $Z$ ) if the question is even (or odd, respectively) and reply with the outcome. Let $h_{j, i}$ be the $i$-th Pauli operator of $h_{j}$. The value this strategy achieves is

$$
\begin{aligned}
\omega_{\mathrm{SPS}}^{*} & =\underset{j}{\mathbb{E}} \frac{1+(-1)^{s_{j}}\left\langle\psi^{\prime}\left|\left(\bigotimes_{i=1}^{5} h_{j, i}\right)\right| \psi^{\prime}\right\rangle}{2} \\
& =\frac{1+\mathbb{E}_{j}\left\langle\psi^{\prime}\left|h_{j}\right| \psi^{\prime}\right\rangle}{2},
\end{aligned}
$$

which gives the desired value using Eq. (14). It is beneficial to restate the above optimal strategy using $|\psi\rangle$ instead of $\left|\psi^{\prime}\right\rangle$. By the conjugation relation between $X, Z$ and $X^{\prime}$ and $Z^{\prime}$, the fifth player essentially measures $X^{\prime}$ and $Z^{\prime}$ on the state $|\psi\rangle$ in the code space when the question is 2,3 respectively. If we think of questions $0,1,2,3$ as measurement specifications of $X, Z, X^{\prime}, Z^{\prime}$, then players who honestly follow the measurement instructions on an encoded state has acceptance probability $\omega_{\mathrm{SPS}}^{*}$. We mention without proof that the classical value of the game is $3 / 4$, again the same as that of the CHSH game.

We have seen a strategy for the game with value $(2+\sqrt{2}) / 4$. The fact that this value is optimal is given in the following theorem. The theorem also proves an important structural result about 
strategies that almost achieve the non-local value of the game. Namely, the special player (the fifth player) must measure honestly the $X^{\prime}$ and $Z^{\prime}$ measurements up to an isometry. It is a partial rigidity property of the special-player stabilizer games.

Theorem 11. Let $\mathscr{S}=\left(\rho,\left\{R_{w}^{(i)}\right\}\right)$ be a strategy for the special-player stabilizer game in Fig. 4 for the five-qubit code, where $\rho$ is the state shared between the players before the game starts and $R_{w}^{(i)}$ is the reflection on Hilbert space $\mathscr{H}_{i}$ describing the projective measurements the player $(i)$ performs when receiving question $w \in\{0,1,2,3\}$. Then the value of the strategy $\mathscr{S}$ is at most $\omega_{S P S}^{*}$ given in Eq. (15). Furthermore, if the value is at least $\omega_{S P S}^{*}-\epsilon$, then there exists an isometry $V \in$ $\mathrm{L}\left(\mathscr{H}_{5}, \mathscr{B} \otimes \hat{\mathscr{H}}_{5}\right)$ such that $R_{3}^{(5)}=V^{\dagger}(Z \otimes I) V$, and

$$
d_{\rho}\left(R_{2}^{(5)}, V^{\dagger}(X \otimes I) V\right) \leq O(\sqrt{\epsilon}) .
$$

We note that some previous works use different distance measures for measurements in the statement of rigidity theorem. For example, the quantity $\|(R-S) \otimes I|\psi\rangle\rangle$ is used in [56] for reflections $R, S$ on state $|\psi\rangle$. It is easy to verify that this is the same as our distance measure $d_{\rho}(R, S)$ up to a constant for $\rho=|\psi\rangle\langle\psi|$.

The proof of the above theorem relies on the following lemmas.

Lemma 12 (Jordan's Lemma [32]). For any two reflections $R_{0}, R_{1}$ acting on a finite dimensional Hilbert space $\mathscr{H}$, there exists a decomposition of $\mathscr{H}$ into orthogonal one- and two-dimensional subspaces invariant under both $R_{0}$ and $R_{1}$.

Lemma 13. Let $R$ be a reflection and $H$ be a Hermitian matrix. Then

$$
\left|\operatorname{tr}_{\rho}(R \otimes H)\right| \leq \operatorname{tr}_{\rho}|H|
$$

Proof. We first prove that

$$
\operatorname{tr}_{\rho}(R \otimes H) \leq \operatorname{tr}_{\rho}|H| .
$$

Let $\mathrm{H}^{+}$and $\mathrm{H}^{-}$be the positive and negative part of $H=\mathrm{H}^{+}-\mathrm{H}^{-}$for positive semidefinite $\mathrm{H}^{+}$ and $H^{-}$. The claim follows by a direct calculation

$$
\begin{aligned}
& \operatorname{tr}_{\rho}(R \otimes H)-\operatorname{tr}_{\rho}|H| \\
= & \operatorname{tr}_{\rho}\left(R \otimes\left(H^{+}-H^{-}\right)\right)-\operatorname{tr}_{\rho}\left(I \otimes\left(H^{+}+H^{-}\right)\right) \\
= & -\operatorname{tr}_{\rho}\left((I-R) \otimes H^{+}\right)-\operatorname{tr}_{\rho}\left((I+R) \otimes H^{-}\right) \leq 0 .
\end{aligned}
$$

A similar argument establishes

$$
\operatorname{tr}_{\rho}(R \otimes H) \geq-\operatorname{tr}_{\rho}|H|
$$

which completes the proof.

Lemma 14. For $\theta_{l} \in[0, \pi], C_{l}=\cos \theta_{l}, S_{l}=\sin \theta_{l}$, and any probability distribution over $l$, if

$$
\underset{l}{\mathbb{E}}\left(\sqrt{1+C_{l}}-1\right)^{2} \leq \epsilon,
$$

then

$$
\underset{l}{\mathbb{E}} S_{l} \geq 1-O(\epsilon)
$$


Proof. Let $\epsilon_{l}$ be $\left(\sqrt{1+C_{l}}-1\right)^{2}$. Then $\mathbb{E}_{l} \epsilon_{l} \leq \epsilon$. For each $l, \epsilon_{l} \leq 1$. We claim that for all $l, S_{l} \geq 1-9 \epsilon_{l}$. This will obviously finish the proof.

As

$$
-2 \sqrt{\epsilon_{l}} \leq C_{l}=\epsilon_{l} \pm 2 \sqrt{\epsilon_{l}} \leq 3 \sqrt{\epsilon_{l}},
$$

it follows that $C_{l}^{2} \leq 9 \epsilon_{l}$. If $\epsilon_{l} \geq 1 / 9$, the claim is trivial since $\theta_{l} \in[0, \pi]$ and $S_{l} \geq 0$. Otherwise,

$$
S_{l}=\sqrt{1-C_{l}^{2}} \geq \sqrt{1-9 \epsilon_{l}} \geq 1-9 \epsilon_{l} .
$$

Proof of Theorem 11. We first give an expression of the game value for the strategy $\mathscr{S}$. For each operator $h_{j}$ in Fig. 3a, define $h_{j}(\mathscr{S})$ as the operator obtained by substituting the $X, Z$ operators in $h_{j}$ with the players' corresponding reflections in the strategy $\mathscr{S}$. That is

$$
h_{j}(\mathscr{S})=(-1)^{s_{j}} \bigotimes_{i=1}^{5} R_{w_{j, i}}^{(i)}
$$

where $s_{j}, w_{j, i}$ are defined in Fig. 4 and $R_{*}^{(i)}$ is defined to be $I$. Following the similar steps as in Eq. (16), the value of $\mathscr{S}$ is computed as

$$
\omega^{*}(\mathscr{S})=\frac{1+\mathbb{E}_{j} \operatorname{tr}_{\rho}\left(h_{j}(\mathscr{S})\right)}{2} .
$$

Consider four matrices

$$
\begin{aligned}
& \Delta_{1}(\mathscr{S})=I \otimes R_{0}^{(2)} \otimes R_{1}^{(3)} \otimes R_{1}^{(4)} \otimes I-I^{\otimes 4} \otimes \frac{R_{2}^{(5)}+R_{3}^{(5)}}{\sqrt{2}}, \\
& \Delta_{2}(\mathscr{S})=R_{0}^{(1)} \otimes I \otimes R_{0}^{(3)} \otimes R_{1}^{(4)} \otimes I-I^{\otimes 4} \otimes \frac{R_{2}^{(5)}-R_{3}^{(5)}}{\sqrt{2}}, \\
& \Delta_{3}(\mathscr{S})=R_{1}^{(1)} \otimes R_{0}^{(2)} \otimes I \otimes R_{0}^{(4)} \otimes I-I^{\otimes 4} \otimes \frac{R_{2}^{(5)}-R_{3}^{(5)}}{\sqrt{2}}, \\
& \Delta_{4}(\mathscr{S})=R_{1}^{(1)} \otimes R_{1}^{(2)} \otimes R_{0}^{(3)} \otimes I \otimes I-I^{\otimes 4} \otimes \frac{R_{2}^{(5)}+R_{3}^{(5)}}{\sqrt{2}} .
\end{aligned}
$$

In the following, we write them as $\Delta_{l}$ and hide their dependence on the strategy $\mathscr{S}$ when there is no ambiguity. The sum of square of the four matrices is

$$
\sum_{l=1}^{4} \Delta_{l}^{2}=8 I-\sqrt{2} \sum_{j} h_{j}(\mathscr{S})
$$

This gives the following expression for the game value

$$
\omega^{*}(\mathscr{S})=\frac{1}{2}+\frac{8 \sqrt{2}-\sqrt{2} \sum_{l=1}^{4} \operatorname{tr}_{\rho}\left(\Delta_{l}^{2}\right)}{32},
$$

from which the optimality of $\omega_{\mathrm{SPS}}^{*}$ is obvious. It also implies that for any strategy $\mathscr{S}$ having value at least $\omega_{\mathrm{SPS}}^{*}-\epsilon$,

$$
\operatorname{tr}_{\rho}\left(\Delta_{1}^{2}\right) \leq \sum_{l=1}^{4} \operatorname{tr}_{\rho}\left(\Delta_{l}^{2}\right) \leq O(\epsilon)
$$


For simplicity, let $R_{2}, R_{3}$ be the shorthand notion of $R_{2}^{(5)}$ and $R_{3}^{(5)}$ in the rest of the proof. Following a similar truncation argument as in [56], we may assume without loss of generality that the underlying Hilbert spaces of the players are finite dimensional so that Jordan's Lemma applies. Using Jordan's lemma and adding extra dimensions if necessary, one get simultaneous 2-by-2 block diagonalizations of $R_{2}$ and $R_{3}$ such that each 2-by-2 block is a reflection having both \pm 1 eigenvalues. Hence, there is an isometry $V \in \mathrm{L}\left(\mathscr{H}_{5}, \mathscr{B} \otimes \hat{\mathscr{H}}_{5}\right)$ such that

$$
R_{3}=V^{\dagger}(Z \otimes I) V,
$$

and

$$
R_{2}=V^{\dagger} \sum_{l}\left[\left(\begin{array}{cc}
C_{l} & S_{l} \\
S_{l} & -C_{l}
\end{array}\right) \otimes|l\rangle\langle l|\right] V
$$

where $C_{l}=\cos \theta_{l}, S_{l}=\sin \theta_{l}$ for $\theta_{l} \in[0, \pi]$ and $l$ is the index of the two-dimensional invariant subspaces obtained by Jordan's lemma.

Substitute the expression for $R_{2}$ and $R_{3}$ in Eq. (18),

$$
\begin{aligned}
O(\epsilon) \geq \operatorname{tr}_{\rho}\left(\Delta_{1}^{2}\right) & =2+\frac{1}{2} \operatorname{tr}_{\rho}\left(R_{2} R_{3}+R_{3} R_{2}\right)+\sqrt{2} \operatorname{tr}_{\rho}\left(R \otimes\left(R_{2}+R_{3}\right)\right) \\
& \geq 2+\frac{1}{2} \operatorname{tr}_{\rho}\left(R_{2} R_{3}+R_{3} R_{2}\right)-\sqrt{2} \operatorname{tr}_{\rho}\left|R_{2}+R_{3}\right| \\
& =2+\frac{1}{2} \operatorname{tr}_{\tilde{\rho}}\left[\sum_{l}\left(\begin{array}{cc}
2 C_{l} & 0 \\
0 & 2 C_{l}
\end{array}\right) \otimes|l\rangle\langle l|\right]-2 \operatorname{tr}_{\tilde{\rho}}\left(\sum_{l} \sqrt{1+C_{l}} I \otimes|l\rangle\langle l|\right) \\
& =\underset{l}{\mathbb{E}}\left(\sqrt{1+C_{l}}-1\right)^{2},
\end{aligned}
$$

where $R$ is the reflection $I \otimes R_{0}^{(2)} \otimes R_{1}^{(3)} \otimes R_{1}^{(4)}$, the second line follows from Lemma 13, $\tilde{\rho}=V \rho V^{\dagger}$, and the expectation $\mathbb{E}_{l}$ is over the probability distribution $\operatorname{Pr}(l)=\operatorname{tr}_{\tilde{\rho}}(I \otimes|l\rangle\langle l|)$.

To complete the proof, consider the state dependent distance between reflections $R_{2}$ and $V^{\dagger}(X \otimes I) V$

$$
\begin{aligned}
d_{\rho}\left(R_{2}, V^{\dagger}(X \otimes I) V\right) & =\left[1-\operatorname{Retr}_{\rho}\left(R_{2} V^{\dagger}(X \otimes I) V\right)\right]^{\frac{1}{2}} \\
& =\left(1-\underset{l}{\mathbb{E}} S_{l}\right)^{\frac{1}{2}} .
\end{aligned}
$$

This equation and Eq. (19) together with Lemma 14 give the second part in the theorem.

In the above discussion, the fifth player plays the role of the special player in the game. It is natural to generalize this to a game with player $(t)$ as the special player. The five-qubit code game with special player $(t)$ is the game defined by the table in Fig. 3b after a cyclic rotation of the question columns such that the special column becomes the $t$-th one. This makes use of the translation invariance of the five-qubit code.

For a general $r$-qubit stabilizer $\mathfrak{S}$ that has a set of $X Z$-form generators $g_{1}, g_{2}, \ldots, g_{l}$, we need to select two generators $g_{X}^{(t)}, g_{Z}^{(t)}$ among $g_{1}, \ldots, g_{l}$ for each qubit $t \in[r]$ such that $g_{X}^{(t)}$ has $X$ operator on the $t$-th qubit and $g_{Z}^{(t)}$ has $Z$ operator on the $t$-th qubit. This is possible in most cases as long as the distance of the stabilizer is larger than or equal to 2 and the $t$-th qubit is not fixed to a pure state for all code states. We call a stabilizer non-trivial if such choices of a pair of generators are possible for all $t \in[r]$. The choice of $g_{X}^{(t)}$ and $g_{Z}^{(t)}$ may not be unique, but any such choice will work. To play the special player stabilizer game with special player $(t)$, we follow the procedure in Fig. 4 with generators $g_{X}^{(t)}$ and $g_{Z}^{(t)}$. Even though the game may not essentially depend on all the generators, the proof of partial rigidity still follows in this general case. 
More specifically, for $t \in[r]$, let $g_{X}^{(t)}$ and $g_{Z}^{(t)}$ be generators that have $X$ and $Z$ on the $t$-th qubit respectively. Following the idea in the design of the game for the five-qubit code, define four operators obtained by doing the $\pi / 4$-trick on the $t$-th qubit to this pair of generators

$$
h_{1}^{(t)}=g_{X}^{(t)}, \quad h_{2}^{(t)}=g_{X \mapsto Z}^{(t)}, \quad h_{3}^{(t)}=g_{Z \mapsto X}^{(t)}, \quad h_{4}^{(t)}=-g_{Z}^{(t)},
$$

where $g_{X \mapsto Z}^{(t)}$ is the operator obtained by changing the $X$ operator of the $t$-th qubit of $g_{X}^{(t)}$ to $Z$ and, similarly, $g_{Z \mapsto X}^{(t)}$ is the operator obtained by changing the $Z$ on $t$-th qubit of $g_{Z}^{(t)}$ to $X$.

As in the case for the five-qubit code game, we translate the operators $h_{j}^{(t)}$ to questions $w_{j}=$ $\left(w_{j, i}\right)$ where $w_{j, i}$ is in $\{*, 0,1,2,3\}$. Following the translation rule, $w_{j, i}$ is $*$ if the $i$-th tensor factor of $h_{j}$ is $I$. It is 0,1 if $i \neq t$ and the corresponding tensor factor is $X, Z$ respectively, and it is 2, 3 if $i=t$ and the tensor factor is $X, Z$ respectively. Similarly, define $s_{j}$ to be 0 or 1 if the sign of $h_{j}^{(t)}$ is 1 or -1 respectively.

The stabilizer game with special player $(t)$ is the game defined as in Fig. 5.

Special-Player Stabilizer Game (General Case)

For a stabilizer $\mathfrak{S}$ with $X Z$-form generators, let $w_{j, i}$ and $s_{j}$ be the questions and parities defined by the operators $h_{j}^{(t)}$ in Eq. (20) for $j \in$ [4]. In the special-player stabilizer game with player $(t)$ as the special player, the verifier performs the following steps:

1. Select an index $j \in[4]$ uniformly at random.

2. For $i \in[r]$, send $w_{j, i}$ in Fig. $3 \mathrm{~b}$ to the player $(i)$ if $w_{j, i}$ is not $*$, the null question.

3. Receive a bit $a^{(i)}$ from player (i) if she was asked a question.

4. Accept if and only if the parity of the answers $\bigoplus_{i} a^{(i)}$ equals $s_{j}$.

Figure 5: Special-player stabilizer game.

\subsection{Stabilizer Game}

The partial rigidity of the special-player stabilizer game applies only to the measurements preformed by the special player. It essentially forces the special player to measure $X^{\prime}$ and $Z^{\prime}$ on her system. There are, however, no rigidity known for the other players' measurements and nothing is proved about the shared state of the strategy. The stabilizer game uses the special-player stabilizer game as a sub-module to achieve the full rigidity properties for all players.

The specification of the Stabilizer Game is given in Fig. 6. It is defined by the $r$-qubit stabilizer $\mathfrak{S}$ with $X Z$-form generators. It also implicitly depends on a fixed choice of generators $g_{X}^{(t)}, g_{Z}^{(t)}$ for each $t \in[r]$ in order to perform the second test. The game involves $r$ players, each of whom may receive a question of two bits, and is required to answer one bit. The verifier's decision depends only on the parity of some of the answer bits and the game is a generalized XOR game. In this paper, the number of qubits $r$ of the stabilizer is always assumed to be a constant, and it may come in the Big- $O$ notions in the rest of the paper.

The non-local value of the game is

$$
\omega_{\mathrm{S}}^{*}=\frac{1+\omega_{\mathrm{SPS}}^{*}}{2}=\frac{6+\sqrt{2}}{8},
$$


Stabilizer Game

For an $r$-qubit non-trivial stabilizer $\mathfrak{S}$ with $X Z$-form generators $g_{1}, g_{2}, \ldots, g_{l}$, define the stabilizer game of $\mathfrak{S}$ as follows. Let $w_{j, i}$ for $j \in[l], i \in[r]$ be $*, 0$, or 1 if $g_{j}$ has $I, X$, or $Z$ on the $i$-th qubit respectively. Let $s_{j}$ for $j \in[l]$ be the 0,1 if the sign of $g_{j}$ is $1,-1$ respectively. The verifier performs the following two tests with equal probability:

1. Select an index $j \in[l]$ uniformly at random. Send $w_{j, i}$ to the player $(i)$ if $w_{j, i} \neq *$. Receives a bit from each player. Accept if the answers not corresponding to the null questions have the same parity as $s_{j}$. Reject otherwise.

2. Select $t \in[r]$ uniformly at random. Play the special-player stabilizer game with special player $(t)$ in Fig. 5.

Figure 6: Stabilizer game for a stabilizer $\mathfrak{S}$.

which can be achieved by players who share an encoded state of the stabilizer code and measures $X, Z, X^{\prime}, Z^{\prime}$ when receiving $0,1,2,3$, respectively. This value is easily seen to be optimal as it saturates the winning probability in both tests of the stabilizer game.

The stabilizer game has the following rigidity property.

Theorem 15. For any non-trivial $r$-qubit stabilizer with $X Z$-form generators, the stabilizer game in Fig. 6 has the following rigidity property. For any strategy $\mathscr{S}$ of the game specified by Hilbert spaces $\left\{\mathscr{H}_{i}\right\}_{i=1}^{r}$, a state $\rho \in \mathrm{D}\left(\bigotimes_{i=1}^{r} \mathscr{H}_{i}\right)$, and reflections $R_{w}^{(i)}$ on $\mathscr{H}_{i}$ for $i \in[r], w \in\{0,1,2,3\}$, if the value of the strategy is at least $\omega_{S}^{*}-\epsilon$, then, there are isometries $V_{i} \in \mathrm{L}\left(\mathscr{H}_{i}, \mathscr{B} \otimes \hat{\mathscr{H}}_{i}\right)$ for $i \in[r]$, such that the following properties holds

- For all $i \in[r], R_{3}^{(i)}=V_{i}^{\dagger}\left(Z^{\prime} \otimes I\right) V_{i}$ and

$$
\begin{gathered}
d_{\rho}\left(R_{2}^{(i)}, V_{i}^{\dagger}\left(X^{\prime} \otimes I\right) V_{i}\right) \leq O\left(\epsilon^{1 / 2}\right) \\
d_{\rho}\left(R_{1}^{(i)}, V_{i}^{\dagger}(Z \otimes I) V_{i}\right) \leq O\left(\epsilon^{1 / 4}\right) \\
d_{\rho}\left(R_{0}^{(i)}, V_{i}^{\dagger}(X \otimes I) V_{i}\right) \leq O\left(\epsilon^{1 / 4}\right)
\end{gathered}
$$

where $X^{\prime}, Z^{\prime}$ are defined in Eq. (2).

- Let $\Pi$ be the projection to the code space of the stabilizer code of $\mathfrak{S}$, and let $V$ be the isometry $\bigotimes_{i=1}^{r} V_{i}$, then

$$
\left\langle\Pi \otimes I, V \rho V^{\dagger}\right\rangle \geq 1-O\left(\epsilon^{1 / 4}\right),
$$

where $\Pi$ acts on the $r$ qubits, each of which is the first qubit of each player after the application of isometry $V$.

Proof. By the symmetry of the game, it suffices to prove the statement for one of the players, say, the player $(r)$. For simplicity, use $R_{w}$ to represent the reflection $R_{w}^{(r)}$ of player $(r)$. It is easy to see that strategy $\mathscr{S}$ wins the special-player stabilizer game of special player $(r)$ with probability $\omega_{\text {SPS }}^{*}-O(\epsilon)$. By Theorem 11, there exists a isometry $V$ such that $R_{3}=V^{\dagger}(Z \otimes I) V$ and

$$
d_{\rho}\left(R_{2}, V^{\dagger}(X \otimes I) V\right) \leq O\left(\epsilon^{1 / 2}\right)
$$


Taking $V_{r}=(W \otimes I) V$, we get the first two conditions in the first item of the theorem, where $W$ is the reflection defined in Eq. (3).

Next, we prove the claim in Eqs. (21b) and (21c). For any $X Z$-form Pauli operator $g$, define $g(\mathscr{S})$ to be the operator obtained by replacing $X$ with $R_{0}^{(i)}$ and $Z$ with $R_{1}^{(i)}$. Let $R$ be the product of the first $r-1$ tensor factors of $g_{X}^{(r)}(\mathscr{S})$. Here, $g_{X}^{(r)}$ is the chosen generator that has $X$ on the $r$-th qubit in the stabilizer game with special player $(r)$. As the strategy $\mathscr{S}$ has value at least $\omega_{\mathrm{S}}^{*}-\epsilon$, it has value at least $1-O(\epsilon)$ for the first test of the stabilizer game, and therefore,

$$
\operatorname{tr}_{\rho}\left(R \otimes R_{0}\right)=\operatorname{tr}_{\rho}\left(g_{X}^{(r)}(\mathscr{S})\right) \geq 1-O(\epsilon) .
$$

In other words, the reflection $R_{0}$ is $O(\epsilon)$-consistent with $R$ on $\rho$. We emphasize that the reflection $R$ acts on the joint system of the first $r-1$ players. This does not cause any problem as it is only used for our proof and is never actually measured on the joint system.

Consider a new strategy $\hat{\mathscr{S}}$ modified from strategy $\mathscr{S}$ by changing $R_{2}$ in the strategy $\mathscr{S}$ to $V_{r}^{\dagger}\left(X^{\prime} \otimes I\right) V_{r}$. This new strategy has value at least $\omega_{\mathrm{S}}^{*}-O(\sqrt{\epsilon})$ by Lemma 7 . Consider the matrix $\Delta_{1}$ for strategy $\hat{\mathscr{S}}$, as in Eq. (17),

$$
\begin{aligned}
\Delta_{1}(\hat{\mathscr{S}}) & =R \otimes I-I \otimes\left[V_{r}^{\dagger}\left(\frac{X^{\prime}+Z^{\prime}}{\sqrt{2}} \otimes I\right) V_{r}\right], \\
& =R \otimes I-I \otimes V_{r}^{\dagger}(X \otimes I) V_{r} .
\end{aligned}
$$

By a similar argument that gives Eq. (18) and the fact that $\hat{\mathscr{S}}$ has value at least $\omega_{\mathrm{SPS}}^{*}-O(\sqrt{\epsilon})$ in the second part of the game, we have

$$
\operatorname{tr}_{\rho}\left(\Delta_{1}^{2}(\hat{\mathscr{S}})\right) \leq O(\sqrt{\epsilon}) .
$$

This gives

$$
\operatorname{tr}_{\rho}\left(R \otimes V_{r}^{\dagger}(X \otimes I) V_{r}\right) \geq 1-O(\sqrt{\epsilon}),
$$

which proves the $O(\sqrt{\epsilon})$-consistency of $V_{r}^{\dagger}(X \otimes I) V_{r}$ and $R$ on $\rho$.

The Eqs. (22) and (23) and Lemma 10 imply that

$$
d_{\rho}\left(R_{0}, V_{r}^{\dagger}(X \otimes I) V_{r}\right) \leq O\left(\epsilon^{1 / 4}\right) .
$$

This completes the proof for Eq. (21c). A similar argument establishes Eq. (21b).

Finally, to prove the second item of the theorem, consider a strategy $\tilde{\mathscr{S}}$ that uses the same state $\rho$ and reflections

$$
\begin{array}{ll}
R_{0}^{(i)}=V_{i}^{\dagger}(X \otimes I) V_{i}, & R_{2}^{(i)}=V_{i}^{\dagger}\left(X^{\prime} \otimes I\right) V_{i}, \\
R_{1}^{(i)}=V_{i}^{\dagger}(Z \otimes I) V_{i}, & R_{3}^{(i)}=V_{i}^{\dagger}\left(Z^{\prime} \otimes I\right) V_{i} .
\end{array}
$$

By Lemma 7 and the first part of the theorem, strategy $\tilde{\mathscr{S}}$ has value at least $\omega_{S}^{*}-O\left(\epsilon^{1 / 4}\right)$. Hence, it has acceptance probability at least $1-O\left(\epsilon^{1 / 4}\right)$ in the first test of the stabilizer game. This means that

$$
\frac{1+\mathbb{E}_{j} \operatorname{tr}_{\tilde{\rho}}\left(g_{j}\right)}{2}=1-O\left(\epsilon^{1 / 4}\right),
$$

where $j$ is uniformly random over $[l], g_{j}$ 's are generators of the stabilizer, and $\tilde{\rho}=V^{\dagger} \rho V$. This is equivalent to

$$
\operatorname{tr}_{\tilde{\rho}}\left(\sum_{j=1}^{l} g_{j}\right)=l-O\left(\epsilon^{1 / 4}\right)
$$


Operator $\sum_{j=1}^{l} g_{j}$ has eigenvalues in $\{-l,-l+2, \ldots, l-2, l\}$ and $\Pi$ projects to the eigenspace of eigenvalue $l$. Hence

$$
\sum_{j=1}^{l} g_{j} \leq l \Pi+(l-2)(I-\Pi)=(l-2) I+2 \Pi .
$$

This, together with Eq. (24), implies that

$$
\operatorname{tr}_{\tilde{\rho}}(\Pi)=1-O\left(\epsilon^{1 / 4}\right),
$$

which is equivalent to the second part of the theorem.

\subsection{Multi-Qubit Stabilizer Game}

In this section, we consider a multi-qubit variant of the stabilizer game called the $(k, n)$-stabilizer game. It is a non-local game implementation of the stabilizer check of the Fitzsimons-Vidick protocol [23]. Instead of asking for the qubits and performing encoding check on them, the verifier sends the measurement instructions on the corresponding qubits to the players. The optimal strategy of the game is to encode each qubit with the stabilizer code and measure honestly the $X, Z, X^{\prime}$ and $Z^{\prime}$ on the encoded data on corresponding qubits. We prove a partial rigidity theorem for the multi-qubit stabilizer game, which suffices for our purpose. In particular, we only prove the rigidity for the reflections corresponding to questions in 0,1 . The full rigidity properties can be proved with some little extra effort.

The $(k, n)$-stabilizer game is given in Fig. 7 . For simplicity, we assume in the multi-qubit stabilizer game that, when the question is $*$, the verifier replaces it with either 0 or 1 and ignore the corresponding answer. With this convention, each player will either see a question of the form $(u, w)$ for $u \in[n]$ and $w=0,1,2,3$ or a tuple of $k$ such questions. Answers are either a single bit or a string of $k$-bits correspondingly. The verifier accepts or rejects depending on the parity of some of the answer bits.

It is easy to see that the non-local value $\omega_{\mathrm{MQS}}^{*}$ of the $(k, n)$-stabilizer game in Fig. 7 equals to the value of the stabilizer game $\omega_{\mathrm{S}}^{*}$. Let $\mathscr{H}_{i}$ be the state space of player $(i)$. A strategy for the $k$-qubit stabilizer game,

$$
\mathscr{S}=\left(\rho,\left\{R_{q}^{(i)}\right\},\left\{M_{\vec{q}}^{(i)}\right\}\right),
$$

consists of a state $\rho \in \mathrm{D}\left(\otimes_{i=1}^{r} \mathscr{H}_{i}\right)$, reflections $R_{q}^{(i)}$ the players measure for question $q$ and measurements $M_{\vec{q}}^{(i)}$ with $k$-bit outcomes for question $\vec{q}$. The superscripts of the measurements indexing the players are sometimes omitted if there will be no ambiguity.

Without loss of generality, it is assumed that the measurements $M_{\vec{q}}$ are projective measurements. For each $q$ that occurs as the $i$-th entry in the tuple $\vec{q}$, define a reflection

$$
S_{q \mid \vec{q}}=\sum_{b \in\{0,1\}^{k}}(-1)^{b_{i}} M_{\vec{q}}^{b}
$$

For $\vec{q}=\left(q_{1}, q_{2}, \ldots, q_{k}\right)$, the measurement $M_{\vec{q}}$ has a one-to-one correspondence with the collection of $k$ pairwise commuting reflections

$$
\left\{S_{q_{s} \mid \vec{q}}\right\}_{s=1}^{k},
$$

and we refer to these reflections as the reflections associated with the projective measurement $M_{\vec{q}}$. We also write $S_{q \mid \vec{q}}$ as $S_{u, w \mid \vec{q}}$ for $q=(u, w)$. On the other hand, for any collection of $k$ pairwise commuting reflections, there associates a projective measurement with $k$-bit outcome as the repeated application of the two-outcome measurements defined by the reflections.

We prove the following partial rigidity property of the $(k, n)$-stabilizer game. 
Multi-Qubit Stabilizer Game

Let $\mathfrak{S}$ be a non-trivial $r$-qubit stabilizer with a set of generators of $X Z$-form. Let $[n]$ be the index of $n$ qubits and let $k \geq 2$ be a constant. The $(k, n)$-stabilizer game for $\mathfrak{S}$ is an $r$-player non-local game where the verifier does the following with equal probability:

1. Select a subset $J \subset[n]$ of size $k$, an index $u \in J$, and a player $t \in[r]$, all uniformly at random. For each qubit $v \in J$, randomly select questions $w_{v}=\left(w_{v, i}\right)$, where $w_{v, i} \in[0,1,2,3]$ and each $w_{v}$ is sampled as in the stabilizer game. Define $q_{v}^{(i)}=$ $\left(\nu, w_{\nu, i}\right)$ for $i \in[r]$ and $v \in J$. Send $q_{u}^{(i)}$ to player $(i)$ and receive an answer bit $a^{(i)}$ if $i \neq t$. Send $\vec{q}=\left(q_{v}^{(t)}\right)_{v \in J}$ to player $(t)$, and receive a $k$-bit string $b=\left(b_{v}\right)_{v \in J}$. Define $a^{(t)}=b_{u}$ and $a=\left(a^{(1)}, a^{(2)}, \ldots, a^{(r)}\right)$. The verifier accepts if and only if the verifier for the stabilizer game accepts when the questions are $w_{u}$ and answers are $a$.

2. Select a qubit $u \in[n]$ uniformly at random. Play the stabilizer game on qubit $u$. That is, the verifier sample $w=\left(w_{i}\right)$ as in the stabilizer game. Define $q^{(i)}=\left(u, w_{i}\right)$ for $i \in[r]$. Send $q^{(i)}$ to player $(i)$ and receive an answer bit $a^{(i)}$. The verifier accepts if the verifier for the stabilizer game accepts on questions $w$ and answers $a=\left(a^{(i)}\right)$.

Figure 7: Multi-qubit stabilizer game.

Theorem 16. For any constant integer $k \geq 2$, there exists a constant $k>0$ that depends only on $k$ such that the $(k, n)$-stabilizer game in Fig. 7 has the following rigidity property. For any quantum strategy $\mathscr{S}=\left(\rho,\left\{R_{q}^{(i)}\right\},\left\{M_{\vec{q}}^{(i)}\right\}\right)$ that has value at least $\omega_{S}^{*}-\epsilon$, there are isometries $V_{i} \in \mathrm{L}\left(\mathscr{H}_{i}, \mathscr{B}^{\otimes n} \otimes\right.$ $\left.\hat{\mathscr{H}}_{i}\right)$, such that the following properties hold

- For all $i \in[r]$, all $q=(u, w), q_{s}=\left(u_{s}, w_{s}\right)$ with $u, u_{s} \in[n], w, w_{s} \in\{0,1\}, s \in[k]$, and $\vec{q}=$ $\left(q_{1}, q_{2}, \ldots, q_{k}\right)$,

$$
\begin{aligned}
d_{\rho}\left(R_{q}^{(i)}, V_{i}^{\dagger} D_{q} V_{i}\right) & \leq O\left(n^{\kappa} \epsilon^{1 / \kappa}\right), \\
d_{\rho}\left(M_{\vec{q}}^{(i)}, N_{\vec{q}}^{(i)}\right) & \leq O\left(n^{\kappa} \epsilon^{1 / \kappa}\right),
\end{aligned}
$$

where $D_{q}$ is the $X, Z$ operator on the $u$-th qubit for $w=0,1$ respectively, and $N_{\vec{q}}^{(i)}$ is the projective measurement with $k$-bit outcome associated with the reflections $\left\{V_{i}^{\dagger} D_{q_{s}} V_{i}\right\}_{s=1}^{k}$.

- Let $\Pi$ be the projection to the code space of the stabilizer code, $V$ be the isometry $\otimes_{i=1}^{r} V_{i}$, then

$$
\left\langle\Pi^{\otimes n} \otimes I, V \rho V^{\dagger}\right\rangle \geq 1-O\left(n^{\kappa} \epsilon^{1 / \kappa}\right),
$$

where the $t$-th tensor factor of $\Pi^{\otimes n}$ acts on $r$ qubits, each of which is the $t$-th qubit of each player's system after the application of $V$.

The proof of Theorem 16 relies on the following lemmas.

Lemma 17. Let $\rho \in \mathrm{D}\left(\mathscr{H}_{A} \otimes \mathscr{H}_{B}\right)$ be a state on systems $A$ and $B$. Let $M_{0}, M_{1}, N_{0}, N_{1}$ be four projective measurements on $\mathscr{H}_{A}$ such that $M_{1}^{a}, N_{1}^{a}$ commute for all a. Let $M, N$ be two projective 
measurements on $\mathscr{H}_{B}$. Suppose that $M_{0}, M_{1}$ are both $\epsilon$-consistent with $M$, and $N_{0}, N_{1}$ are both $\epsilon$-consistent with $N$ on state $\rho$. Then

$$
\sum_{a}\left\|\left[M_{0}^{a}, N_{0}^{a}\right]\right\|_{\rho}^{2} \leq O(\sqrt{\epsilon}) .
$$

Proof. First prove that

$$
\sum_{a} \operatorname{tr}_{\rho}\left(M_{0}^{a} N_{0}^{a} M_{0}^{a} N_{0}^{a}\right) \approx{ }_{\sqrt{\epsilon}} \sum_{a} \operatorname{tr}_{\rho}\left(N_{0}^{a} M_{0}^{a} N_{0}^{a} M_{1}^{a}\right) .
$$

Namely, we can move operator $M_{0}^{a}$ in the front to the end of the product and change it to $M_{1}^{a}$ without incurring too much error in the expression.

By $\epsilon$-consistency between $M_{0}$ and $M$,

$$
\sum_{a} \operatorname{tr}_{\rho}\left(M_{0}^{a} N_{0}^{a} M_{0}^{a} N_{0}^{a}\right) \approx \sqrt{\epsilon} \sum_{a} \operatorname{tr}_{\rho}\left(M_{0}^{a} N_{0}^{a} M_{0}^{a} N_{0}^{a} \otimes M^{a}\right),
$$

as the absolute value of the difference on the two sides is

$$
\begin{aligned}
& \left|\sum_{a} \operatorname{tr}_{\rho}\left(M_{0}^{a} N_{0}^{a} M_{0}^{a} N_{0}^{a} \otimes\left(1-M^{a}\right)\right)\right| \\
\leq & \sqrt{\sum_{a} \operatorname{tr}_{\rho}\left(M_{0}^{a} \otimes\left(1-M^{a}\right)\right) \sum_{a} \operatorname{tr}_{\rho}\left(N_{0}^{a} M_{0}^{a} N_{0}^{a} M_{0}^{a} N_{0}^{a}\right)} \\
\leq & \sqrt{\epsilon} .
\end{aligned}
$$

By similar arguments,

$$
\begin{aligned}
\sum_{a} \operatorname{tr}_{\rho}\left(M_{0}^{a} N_{0}^{a} M_{0}^{a} N_{0}^{a} \otimes M^{a}\right) & \approx_{\sqrt{\epsilon}} \sum_{a} \operatorname{tr}_{\rho}\left(N_{0}^{a} M_{0}^{a} N_{0}^{a} \otimes M^{a}\right) \\
& \approx_{\sqrt{\epsilon}} \sum_{a} \operatorname{tr}_{\rho}\left(N_{0}^{a} M_{0}^{a} N_{0}^{a} M_{1}^{a} \otimes M^{a}\right) \\
& \approx_{\sqrt{\epsilon}} \sum_{a} \operatorname{tr}_{\rho}\left(N_{0}^{a} M_{0}^{a} N_{0}^{a} M_{1}^{a}\right) .
\end{aligned}
$$

This proves our claim about moving and changing $M_{0}^{a}$ to $M_{1}^{a}$. Do this for the four operators in the product sequentially,

$$
\sum_{a} \operatorname{tr}_{\rho}\left(M_{0}^{a} N_{0}^{a} M_{0}^{a} N_{0}^{a}\right) \approx{ }_{\sqrt{\epsilon}} \sum_{a} \operatorname{tr}_{\rho}\left(M_{1}^{a} N_{1}^{a} M_{1}^{a} N_{1}^{a}\right) .
$$

Expand the $\rho$-norm in left hand side of the claim in the lemma,

$$
\sum_{a}\left\|\left[M_{0}^{a}, N_{0}^{a}\right]\right\|_{\rho}^{2}=\sum_{a} \operatorname{tr}_{\rho}\left[M_{0}^{a} N_{0}^{a} M_{0}^{a}+N_{0}^{a} M_{0}^{a} N_{0}^{a}-M_{0}^{a} N_{0}^{a} M_{0}^{a} N_{0}^{a}-N_{0}^{a} M_{0}^{a} N_{0}^{a} M_{0}^{a}\right] .
$$

Now the proof follows by applying the above operator moving procedure to each of the four terms in the expansion and the condition that $M_{1}^{a}$ commutes with $N_{1}^{a}$ for all $a$.

Lemma 18. Let $\mathscr{B}_{1}, \mathscr{B}_{1^{\prime}}, \mathscr{B}$ be two-dimensional Hilbert spaces. Let $V \in \mathrm{L}(\mathscr{H}, \mathscr{B} \otimes \hat{\mathscr{O}})$ be an isometry, $R \in \mathrm{L}(\mathscr{H})$ be an operator and $|\Phi\rangle$ be the EPR state on $\mathscr{B}_{1} \otimes \mathscr{B}_{1^{\prime}}$. Define isometry $C \in$ $\mathrm{L}\left(\not{H}, \mathscr{B}_{1} \otimes \mathscr{B}_{1^{\prime}} \otimes \mathscr{H}\right)$ as

$$
C=\left(I \otimes V^{\dagger}\right) \operatorname{SWAP}(|\Phi\rangle \otimes V),
$$

where the SWAP acts on $\mathscr{B}_{1}$ and $\mathscr{B}$. Then

$$
C^{\dagger} R C=\underset{j=0,1,2,3}{\mathbb{E}}\left(V^{\dagger} \sigma_{j} V\right) R\left(V^{\dagger} \sigma_{j} V\right),
$$

where $\sigma_{j}$ are Pauli operators. 
Proof. This follows by substituting the two SWAP gates using the identity

$$
\mathrm{SWAP}=\frac{I+X X+Y Y+Z Z}{2},
$$

and a direct calculation.

Lemma 19. Let $\rho \in \mathrm{D}\left(\mathscr{H} \otimes \mathscr{H}^{\prime}\right)$ be a state, $T \in \mathrm{L}(\mathscr{H})$ be an operator with constant operator norm, $R$ be a reflection on $\mathscr{H}$ that has an $\epsilon$-consistent reflection $S$ on $\mathscr{H}^{\prime}$. Then

$$
\operatorname{tr}_{\rho}(R T R) \approx \sqrt{\epsilon} \operatorname{tr}_{\rho}(T)
$$

Proof. We first prove that

$$
\operatorname{tr}_{\rho}(R T) \approx \sqrt{\epsilon} \operatorname{tr}_{\rho}(T \otimes S)
$$

By consistency of $R$ and $S$, we have

$$
\begin{aligned}
\operatorname{tr}_{\rho}(R T) & =\sum_{a \in\{0,1\}} \operatorname{tr}_{\rho}\left(R^{a}(-1)^{a} T\right) \\
& \approx \sqrt{\epsilon} \sum_{a \in\{0,1\}} \operatorname{tr}_{\rho}\left(R^{a}(-1)^{a} T \otimes S^{a}\right) \\
& \approx \sqrt{\epsilon} \sum_{a \in\{0,1\}} \operatorname{tr}_{\rho}\left((-1)^{a} T \otimes S^{a}\right) \\
& =\operatorname{tr}_{\rho}(T \otimes S)
\end{aligned}
$$

Similarly,

$$
\operatorname{tr}_{\rho}(T R) \approx \sqrt{\epsilon} \operatorname{tr}_{\rho}(T \otimes S)
$$

Taking $T=T R$ in Eqs. (27) and (28), we have $\operatorname{tr}_{\rho}(R T R) \approx{ }_{\sqrt{\epsilon}} \operatorname{tr}_{\rho}(T R \otimes S)$, and

$$
\operatorname{tr}_{\rho}(T)=\operatorname{tr}_{\rho}(T R R) \approx \sqrt{\epsilon} \operatorname{tr}_{\rho}(T R \otimes S)
$$

This completes the proof.

Lemma 20. Let $\rho \in \mathrm{D}\left(\mathscr{H}_{A} \otimes \mathscr{H}_{B}\right)$ be a quantum state of systems $A$ and $B, M=\left\{M^{b}\right\}$ and $N=$ $\left\{N^{b}\right\}$ be two projective measurements with $k$-bit outcomes on system A. Let $\left\{R_{j}\right\}_{j=1}^{k},\left\{S_{j}\right\}_{j=1}^{k}$ be the reflections associated with the projective measurements $M$ and $N$ respectively. If there are reflections $T_{j}$ such that $R_{j}$ and $S_{j}$ are both $\epsilon$-consistent with $T_{j}$ on $\rho$ for all $j=1,2, \ldots, k$, then

$$
d_{\rho}(M, N) \leq O\left(\epsilon^{1 / 4}\right) .
$$

Proof. We prove the case of $k=2$. The general case follows from a similar argument. Using the consistency conditions,

$$
\begin{aligned}
\sum_{b \in\{0,1\}^{2}} \operatorname{tr}_{\rho}\left(M^{b} N^{b}\right) & =\sum_{b_{1}, b_{2} \in\{0,1\}} \operatorname{tr}_{\rho}\left(R_{1}^{b_{1}} R_{2}^{b_{2}} S_{2}^{b_{2}} S_{1}^{b_{1}}\right) \\
& \approx \sqrt{\epsilon} \sum_{b_{1}, b_{2} \in\{0,1\}} \operatorname{tr}_{\rho}\left(R_{1}^{b_{1}} R_{2}^{b_{2}} S_{2}^{b_{2}} S_{1}^{b_{1}} \otimes T_{1}^{b_{1}}\right) \\
& \approx \sqrt{\epsilon} \sum_{b_{1}, b_{2} \in\{0,1\}} \operatorname{tr}_{\rho}\left(R_{2}^{b_{2}} S_{2}^{b_{2}} \otimes T_{1}^{b_{1}}\right) \\
& =\sum_{b_{2} \in\{0,1\}} \operatorname{tr}_{\rho}\left(R_{2}^{b_{2}} S_{2}^{b_{2}}\right) \approx_{\epsilon} 1 .
\end{aligned}
$$

The first approximation uses the consistency condition to add the operator $T_{1}^{b_{1}}$, the second approximation uses the consistency condition to remove operators $R_{1}^{b_{1}}$ and $S_{1}^{b_{1}}$, the last approximation is by Lemma 10. 
Proof of Theorem 16. Consider first the claim in Eq. (25a) of the theorem for $i=r$.

In the proof, $D_{w}$ denotes $X, Z$ for $w=0,1$ respectively, and $D_{q}$ denotes $D_{w}$ acting on the $u$-th qubit if $q=(u, w)$. Let $\delta$ be $n \epsilon$ and $\delta_{k}$ be $n^{k} \epsilon$. For simplicity, we omit the superscript $(r)$ in the reflections for player $(r)$.

Since the strategy $\mathscr{S}$ has value at least $\omega_{\mathrm{S}}^{*}-\epsilon$ for the $(k, n)$-stabilizer game, it must have value at least $\omega_{\mathrm{S}}^{*}-O(\delta)$ for the stabilizer games for each $u \in[n]$ in the second part of the game. More precisely, $\mathscr{S}_{u}=\left(\rho,\left\{R_{u, w}^{(i)}\right\}\right)$ forms a strategy for the stabilizer game with value at least $\omega_{\mathrm{S}}^{*}-O(\delta)$.

By Theorem 15, for all $u \in[n]$, there exist isometries $V_{u} \in \mathrm{L}\left(\mathscr{H}_{r}, \mathscr{B} \otimes \tilde{\mathscr{H}}_{r}\right)$ such that

$$
d_{\rho}\left(R_{u, w}, V_{u}^{\dagger}\left(D_{w} \otimes I\right) V_{u}\right) \leq O\left(\delta^{1 / 4}\right) .
$$

Define $\hat{R}_{u, w}=V_{u}^{\dagger}\left(D_{w} \otimes I\right) V_{u}$. The above equation becomes

$$
d_{\rho}\left(R_{u, w}, \hat{R}_{u, w}\right) \leq O\left(\delta^{1 / 4}\right) .
$$

Similarly, for all $J \subseteq[n]$ and $u \in J$, all choices of $w_{v}$ for $v \in J$ and $v \neq u$, consider state $\rho$, reflections $R_{u, w}^{(i)}$ for $i \in[r-1]$ and $S_{u, w \mid \vec{q}}$ for player $(r)$. They form a strategy for the stabilizer game with value at least $\omega_{\mathrm{S}}^{*}-O\left(\delta_{k}\right)$ for $q_{u}=(u, w), q_{v}=\left(\nu, w_{v}\right)$ and $\vec{q}=\left(q_{v}\right)_{v \in J}$. We clarify that only $w$ is the index of questions for the stabilizer game in this strategy and everything else in the subscripts are fixed.

By Eqs. (22) and (23), reflection $S_{u, w \mid \vec{q}}$ and $\hat{R}_{u, w}$ have the same $O\left(\sqrt{\delta_{k}}\right)$-consistent measurement on $\rho$. Let $(u, w)$ and $\left(\nu, w^{\prime}\right)$ be two entries of $\vec{q}$, then the reflections $\hat{R}_{u, w}, S_{u, w \mid \vec{q}}, S_{v, w^{\prime} \mid \vec{q}}$, $\hat{R}_{\nu, w^{\prime}}$ corresponds to measurements that satisfy the conditions for $M_{0}, M_{1}, N_{1}, N_{0}$ in Lemma 17. Hence,

$$
\left\|\left[\hat{R}_{u, w}, \hat{R}_{v, w^{\prime}}\right]\right\|_{\rho}^{2} \leq O\left(\delta_{k}^{1 / 4}\right)
$$

for all $u \neq v \in[n]$.

Consider $2 n$ two-dimensional Hilbert spaces $\mathscr{B}_{u}, \mathscr{B}_{u^{\prime}}$ for $u \in[n]$. Denote $\mathscr{H}_{[n]}=\bigotimes_{u=1}^{n} \mathscr{B}_{u}$ and $\mathscr{H}_{[n]^{\prime}}=\bigotimes_{u=1}^{n} \mathscr{B}_{u^{\prime}}$. We sometimes call the system of $\mathscr{B}_{u}$ as the $u$-th qubit. Let $|\Phi\rangle_{u, u^{\prime}}$ be the EPR state on systems $\mathscr{B}_{u}, \mathscr{B}_{u^{\prime}}$. For each $u \in[n]$, define isometry $C_{u} \in \mathrm{L}\left(\mathscr{H}, \mathscr{B}_{u} \otimes \mathscr{B}_{u^{\prime}} \otimes \mathscr{H}\right)$ as

$$
C_{u}=\left(I \otimes V_{u}^{\dagger}\right) \operatorname{SWAP}_{u}\left(|\Phi\rangle_{u, u^{\prime}} \otimes V_{u}\right),
$$

where $\mathrm{SWAP}_{u}$ is the SWAP gate acting on the $u$-th qubit and the first output qubit of $V_{u}$.

Define isometry $V \in \mathrm{L}\left(\mathscr{H}_{r}, \mathscr{H}_{[n]} \otimes \mathscr{H}_{[n]^{\prime}} \otimes \mathscr{H}_{r}\right)$ as the sequential application of $C_{1}, C_{2}, \ldots, C_{n}$,

$$
V=C_{n} C_{n-1} \cdots C_{1} \text {. }
$$

We claim that this choice of $V$ works for the claims of the theorem by taking $\hat{\mathscr{H}}_{r}$ to be $\mathscr{H}_{[n]^{\prime}} \otimes \mathscr{H}_{r}$. Define

$$
\tilde{R}_{q}=V^{\dagger} D_{q} V,
$$

for $q=(u, w)$. The aim is to first prove that

$$
d_{\rho}\left(\hat{R}_{q}, \tilde{R}_{q}\right) \leq O\left(n^{\kappa} \epsilon^{1 / \kappa}\right) .
$$

Recall that $\sigma_{s}$ for $s=0,1,2,3$ are the Pauli operators. Define reflection $T_{q}=V_{u}^{\dagger} \sigma_{s} V_{u}$ for $q=$ $(u, s)$ and the corresponding superoperator $\mathscr{T}_{q}(\sigma)=T_{q} \sigma T_{q}$. It is easy to verify that

$$
T_{u, 0}=I, \quad T_{u, 1}=\hat{R}_{u, 0}, \quad T_{u, 2}=-i \hat{R}_{u, 0} \hat{R}_{u, 1}, \quad T_{u, 3}=\hat{R}_{u, 1} .
$$


As $D_{q}$ and $C_{v}$ commutes for all $v>u$ and $q=(u, w)$, we have

$$
\tilde{R}_{q}=C_{1}^{\dagger} C_{2}^{\dagger} \ldots C_{u-1}^{\dagger} \hat{R}_{q} C_{u-1} C_{u-2} \ldots C_{1} .
$$

A series of applications of Lemma 18 gives the expression of $\tilde{R}_{q}$ for $q=(u, w)$,

$$
\tilde{R}_{q}=\underset{s \in\{0,1,2,3\}^{u-1}}{\mathbb{E}} \mathscr{T}_{1, s_{1}} \circ \mathscr{T}_{2, s_{2}} \circ \cdots \circ \mathscr{T}_{u-1, s_{u-1}}\left(\hat{R}_{q}\right) .
$$

For convenience, define

$$
R=\underset{s_{2}, \ldots, s_{u-1}}{\mathbb{E}} \mathscr{T}_{2, s_{2}} \circ \cdots \circ \mathscr{T}_{u-1, s_{u-1}}\left(\hat{R}_{q}\right)
$$

Then

$$
\tilde{R}_{q}=\underset{s_{1} \in\{0,1,2,3\}}{\mathbb{E}} \mathscr{T}_{1, s_{1}}(R),
$$

and

$$
\operatorname{tr}_{\rho}\left(\tilde{R}_{q} \hat{R}_{q}\right)=\underset{s_{1} \in\{0,1,2,3\}}{\mathbb{E}} \operatorname{tr}_{\rho}\left(\mathscr{T}_{1, s_{1}}(R) \hat{R}_{q}\right) .
$$

For each of the four cases for $s_{1}$, it is claimed that $\operatorname{tr}_{\rho}\left(\mathscr{T}_{1, s_{1}}(R) \hat{R}_{q}\right)$ is close $\operatorname{to~}_{\rho}\left(R \hat{R}_{q}\right)$. In words, removing the superoperator $\mathscr{T}_{1, s_{1}}$ induces a bounded error in the expression.

Consider the case $s_{1}=1$ first. In this case

$$
\begin{aligned}
\operatorname{tr}_{\rho}\left(\mathscr{T}_{1,1}(R) \hat{R}_{q}\right) & =\operatorname{tr}_{\rho}\left(\hat{R}_{1,0} R \hat{R}_{1,0} \hat{R}_{q}\right) \\
& \approx_{\delta_{k}^{1 / 8}} \operatorname{tr}_{\rho}\left(\hat{R}_{1,0} R \hat{R}_{q} \hat{R}_{1,0}\right) \\
& \approx_{\delta^{1 / 4}} \operatorname{tr}_{\rho}\left(R \hat{R}_{q}\right),
\end{aligned}
$$

where the first approximation follows from Eq. (30) and Cauchy-Schwarz inequality, the second approximation follows from Lemma 19 and the fact that $\hat{R}_{1,0}$ has an $O\left(\delta^{1 / 2}\right)$-consistency reflection on $\rho$ by Eq. (23).

A similar argument applies for the other cases of $s_{1}$. Repeat this procedure of removing the superoperators $\mathscr{T}_{j, s_{j}}$ one by one, we have

$$
\operatorname{tr}_{\rho}\left(\tilde{R}_{q}, \hat{R}_{q}\right) \approx_{u \delta_{k}^{1 / 8}} \operatorname{tr}_{\rho}\left(\hat{R}_{q}, \hat{R}_{q}\right)=1
$$

and

$$
d_{\rho}\left(\tilde{R}_{q}, \hat{R}_{q}\right) \leq O\left(\sqrt{u \delta_{k}^{1 / 8}}\right) \leq O\left(n^{1 / 2} \delta_{k}^{1 / 16}\right) .
$$

By the triangle inequality of $d_{\rho}$ and Eq. (29)

$$
d_{\rho}\left(\tilde{R}_{q}, R_{q}\right) \leq O\left(n^{1 / 2} \delta_{k}^{1 / 16}\right) .
$$

This proves the bound in Eq. (25a) by choosing $\kappa$ sufficiently large.

Recall that there exists a reflection $R$ that is $\delta_{k}$-consistent with both $R_{q}$ and $S_{q \mid \vec{q}}$ on $\rho$. By the bound in Eq. (33) and Lemma 7,

$$
C_{\rho}\left(R, \tilde{R}_{q}\right) \geq 1-O\left(n^{1 / 2} \delta_{k}^{1 / 16}\right) .
$$

The bounds in Eq. (25b) follows from Lemma 20 and the consistency of reflections $\tilde{R}_{q}, S_{q \mid \vec{q}}$ with the same reflection $R$ on the first $r-1$ players' systems.

The second part of the theorem follows by a similar argument used to prove the second part of Theorem 15. 


\section{Non-Local Games for Local Hamiltonian Problems}

In this section, we give the non-local game for the local Hamiltonian problem. Consider a restricted form of the local Hamiltonian problem in the following definition.

Definition 21. Consider a $k$-local Hamiltonian of $m$ terms on $n$ qubits

$$
H=\sum_{j=1}^{m} H_{j}
$$

where $0 \leq H_{j} \leq I$ acts on at most $k$ qubits. The Hamiltonian $H$ is $X Z$-form if $H_{j}$ is a real linear combination of tensor products of $I, X, Z$ for each $j$.

Lemma 22. There exist constant $k$ such that the $X Z$-form $k$-local Hamiltonian problem is QMAcomplete.

Proof. This is a simple corollary of the results in [12]. The gate set $\{$ CNOT, $X, W=\cos (\pi / 8) X+$ $\sin (\pi / 8) Z$ \} is known to be universal by the result of Shi [58] and each gate $U_{t}$ in the gate set is of $X Z$-form and $U_{t}=U_{t}^{\dagger}$. Start with a circuit using this particular set of gates and perform the circuit to Hamiltonian construction of Kitaev. The 5-local terms resulting from the construction will have the $X Z$-form. For example, the term checking the propagation of the $t$-th step of the circuit is

$$
\begin{aligned}
H_{\text {prop }, t}= & I \otimes|100\rangle\left\langle\left. 100\right|_{t-1, t, t+1}-U_{t} \otimes \mid 110\right\rangle\left\langle\left. 100\right|_{t-1, t, t+1}\right. \\
& -U_{t}^{\dagger} \otimes|100\rangle\left\langle\left. 110\right|_{t-1, t, t+1}+I \otimes \mid 110\right\rangle\left\langle\left. 110\right|_{t-1, t, t+1}\right. \\
= & \frac{I-Z_{(t-1)}}{2} \otimes \frac{I+Z_{(t+1)}}{2}-U_{t} \otimes \frac{I-Z_{(t-1)}}{2} \otimes X_{(t)} \otimes \frac{1+Z_{(t+1)}}{2},
\end{aligned}
$$

which is easily seen to have $X Z$-form for $U_{t}$ in the chosen gate set. Other terms in the construction can be checked similarly. This proves the claim for $k=5$.

Using more advanced results from [20], such as their Lemma 22, one can prove the claim for $k=2$ and the two-local terms $H_{j}$ have the form $\alpha_{j}(X Z-Z X)$.

Let $H=\sum_{j=1}^{m} H_{j}$ be the Hamiltonian and assume that $0 \leq H_{j} \leq I$. We will consider two different types of energy measurements for a $k$-local Hamiltonian $H$ on a state $\rho$. The first type of measurement is the one used in [23] and does the following. The verifier randomly selects $j \in[m]$ and gets the $k$-qubit state $\rho_{j}$ on which $H_{j}$ acts. Then the verifier measures the POVM $\left\{H_{j}, I-H_{j}\right\}$ and rejects when the measurement result is ' $H_{j}$ '. It is easy to see that the verifier rejects with probability

$$
\frac{1}{m} \sum_{j=1}^{m}\left\langle H_{j}, \rho\right\rangle=\frac{1}{m}\langle H, \rho\rangle .
$$

In the second type of energy measurement, we only measure Pauli operators. Let $\mathscr{P}_{X Z}$ be the set of the $3^{k} k$-fold tensor products of $I, X, Z$ operators. For $X Z$-form Hamiltonians, it suffices to measure the Pauli operators in $\mathscr{P}_{X Z}$ only. Expand each term

$$
H_{j}=\sum_{P \in \mathscr{P}_{X Z}} \alpha_{j, P} P .
$$

Computing the trace of squared operators on both sides of Eq. (34), we have

$$
\sum_{P \in \mathscr{P}_{X Z}} \alpha_{j, P}^{2} \leq 1 .
$$


The verifier randomly selects $j \in[m]$ and gets the $k$-qubit state $\rho_{j}$ on which $H_{j}$ acts. He then chooses $P$ uniformly at random and measures $P$ on $\rho_{j}$. The verifier rejects with probability $\left|\alpha_{j, P}\right|$ if either $\alpha_{j, P}>0$ and the measurement result is +1 , or $\alpha_{j, P}<0$ and the measurement result is -1 . The probability of rejection is computed as

$$
\frac{1}{3^{k} m} \sum_{j} \sum_{P} \frac{\left|\alpha_{j, P}\right|+\alpha_{j, P}\left\langle P, \rho_{j}\right\rangle}{2}=\frac{\alpha m+\langle H, \rho\rangle}{2 \cdot 3^{k} m},
$$

where

$$
\alpha=\sum_{j, P}\left|\alpha_{j, P}\right| / m
$$

is a constant determined by the Hamiltonian.

We note that the second type of the energy measurement is less efficient but the probabilities of rejection in these two settings are linearly related. In fact, it is easy to see that the rejection probability in the first setting is $p$ if and only if the rejection probability of the second setting is

$$
\frac{\alpha+p}{2 \cdot 3^{k}}
$$

We now give the non-local game for the local Hamiltonian problem as in Fig. 8. To measure the energy, we further assume that the stabilizer $\mathfrak{S}$ used in the game has the logical $X, Z$ operators $L_{X}$ and $L_{Z}$ that are products of $I, X, Z$. This is the case for both the five-qubit code and the four-qubit quantum error detecting code.

Theorem 23. There exists constants $C$ and $\kappa$ such that the following holds. If $p$ in the $r$-player game for an $X Z$-form, $k$-local Hamiltonian problem in Fig. 8 is chosen to be $C(b-a)^{\kappa} / n^{\kappa}$, and $\alpha$ is defined as in Eq. (35), then

1. For yes-instance of the k-local Hamiltonian problem, the non-local value of the game is at least

$$
(1-p) \omega_{S}^{*}+p\left(1-\frac{\alpha+a}{2 \cdot 3^{k}}\right) .
$$

2. For no-instance of the k-local Hamiltonian problem, the non-local value of the game is at most

$$
(1-p) \omega_{S}^{*}+p\left(1-\frac{\alpha+(a+b) / 2}{2 \cdot 3^{k}}\right) .
$$

Proof. First consider the completeness of the game. If the local Hamiltonian problem is a yesinstance, there exists a quantum witness state $|\psi\rangle \in \mathscr{B}^{\otimes n}$ such that

$$
\langle\psi|H| \psi\rangle \leq a m
$$

We construct the strategy for the $r$ players as follows. For each qubit $u$ of $|\psi\rangle$, we encode it with the stabilizer code $\mathfrak{S}$ and let player $(i)$ hold the $i$-th encoded qubit of $u$. When receiving the question $(u, w)$ from the verifier, the players measure their share of qubit $u$ with $X, Z, X^{\prime}, Z^{\prime}$ correspondingly if $w=0,1,2,3$.

For this strategy, the players can win the Encoding Check part with optimal probability $\omega_{\mathrm{S}}^{*}$. In the Energy Check part of the game, the measurement of the logical $X$ and logical $Z$ is essentially an implementation of the second type of energy measurement on the state $\psi$. The rejection probability in this part is

$$
\frac{\alpha m+\langle H, \mid \psi\rangle\langle\psi \mid\rangle}{2 \cdot 3^{k} m} .
$$


Non-Local Game for The Local Hamiltonian Problem

Let $\mathfrak{S}$ be a non-trivial $r$-qubit stabilizer code with $X Z$-form generators that encodes at least one qubit and has a pair of logical $L_{X}, L_{Z}$ operators of $X Z$-form. Define two question vectors $w_{X}=\left(w_{X, i}\right)$ and $w_{Z}=\left(w_{Z, i}\right)$ as follows. The entry $w_{D, i}$ is $*, 0$, or 1 , if the $i$-th Pauli factor of the logical operator $L_{D}$ is $I, X, Z$ respectively for $D=X, Z$. For an $X Z$-form, $k$-local Hamiltonian problem $(H, a, b)$, and a small probability $p$ chosen later, we consider the following multi-player non-local game. It involves a classical verifier and $r$ players $(i)$ for $i \in[r]$. The verifier performs the first test with probability $p$, and the second test with probability $1-p$ :

1. Energy Check. Select $j \in[m]$ uniformly at random. Expand $H_{j}$ as

$$
H_{j}=\sum_{P \in \mathscr{P}_{X Z}} \alpha_{j, P} P
$$

and let $J \subset[n]$ be the set of $k$ qubits $H_{j}$ acts on non-trivially. Select an operator $P$ in $\mathscr{P}_{X Z}$ uniformly at random. For each $u \in J$, define $w_{u, i}$ to be $w_{X, i}$ or $w_{Z, i}$ if the tensor factor in $P$ acting on qubit $u$ is $X$ or $Z$, respectively. Define $q_{u}^{(i)}=\left(u, w_{u, i}\right)$ and $\vec{q}=\left(q_{u}^{(i)}\right)_{u \in J}$. Send the question $\vec{q}$ to the $r$ players. Receive a $k$-bit answer $a^{(i)}=\left(a_{u}^{(i)}\right)$ from each player. The verifier rejects with probability $\left|\alpha_{j, P}\right|$ if either the parity of the answer bits not corresponding to the null question is even and $\alpha_{j, P}>0$, or the parity is odd and $\alpha_{j, P}<0$.

2. Encoding Check. Play the $(k, n)$-stabilizer game.

Figure 8: The non-local game for local Hamiltonian problems

Therefore, the acceptance probability of the game $\omega^{*}$ is at least

$$
(1-p) \omega_{\mathrm{S}}^{*}+p\left(1-\frac{\alpha+a}{2 \cdot 3^{k}}\right) \text {. }
$$

Next, we prove the soundness of the game. If the local Hamiltonian problem defined by $(H, a, b)$ is a no-instance, we need to prove an upper bound of the non-local value of the game.

Consider any strategy $\mathscr{S}$ that has acceptance probability $\omega_{\mathrm{S}}^{*}-\epsilon$ in the Encoding Check part of the game. Theorem 16 states that there are isometries $V_{i} \in \mathrm{L}\left(\mathscr{H}_{i}, \mathscr{B}^{\otimes n} \otimes \hat{\mathscr{H}}_{i}\right)$, measurements $N_{\vec{q}}$ associated with $\left\{V_{i}^{\dagger} D_{q_{s}} V_{i}\right\}_{s=1}^{k}$, such that

$$
d_{\rho}\left(M_{\vec{q}}, N_{\vec{q}}\right) \leq O\left(n^{\kappa} \epsilon^{1 / \kappa}\right) .
$$

Consider the strategy $\tilde{\mathscr{S}}=\left(\rho,\left\{R_{q}\right\},\left\{N_{\vec{q}}\right\}\right)$. The value of $\mathscr{S}$ and $\tilde{\mathscr{S}}$ for the Energy Check differ at most by $O\left(n^{\kappa} \epsilon^{1 / \kappa}\right)$ by Lemma 7 .

Strategy $\tilde{\mathscr{S}}$ uses honest $X, Z$ measurement on the logical space of the error correcting code and we claim that it must have value at most

$$
1-\frac{\alpha+b}{2 \cdot 3^{k}}
$$

in the Energy Check part. Otherwise, the state of the first $r n$ qubits after the application of $\otimes_{i} V_{i}$ has rejection probability at most $(\alpha+b) /\left(2 \cdot 3^{k}\right)$ in the energy measurement using logical $X, Z$ 
operators $L_{X}, L_{Z}$. This implies the existence of $n$-qubit state that has rejection probability at most $(\alpha+b) /\left(2 \cdot 3^{k}\right)$ in the second type energy measurement, which is a contradiction to the no-instance condition of the local Hamiltonian problem.

Therefore, the value of strategy $\mathscr{S}$ is at most

$$
(1-p)\left(\omega_{\mathrm{S}}^{*}-\epsilon\right)+p\left(1-\frac{\alpha+b}{2 \cdot 3^{k}}+c n^{\kappa} \epsilon^{1 / \kappa}\right),
$$

for constants $c, \kappa$ large enough.

Maximizing the expression as a function of $\epsilon$, it is easy to see that the maximum value is achieved at

$$
\epsilon=\left(\frac{p c n^{\kappa}}{(1-p) \kappa}\right)^{\kappa /(\kappa-1)}
$$

Substituting this into Eq. (36), $\omega^{*}(\mathscr{S})$ is upper bounded by

$$
(1-p) \omega_{\mathrm{S}}^{*}+p\left(1-\frac{\alpha+b}{2 \cdot 3^{k}}+\Delta\right)
$$

for

$$
\Delta=\left(1-\frac{1}{\kappa}\right) c n^{\kappa}\left(\frac{p c n^{\kappa}}{(1-p) \kappa}\right)^{1 /(\kappa-1)} .
$$

Choosing $\kappa$ large and $p$ small such that $(1-p) \kappa \geq 1$, we can bound $\Delta$ as

$$
\Delta \leq c n^{\kappa}\left(p c n^{\kappa}\right)^{1 /(\kappa-1)}=\left(p c^{\kappa} n^{\kappa^{2}}\right)^{1 /(\kappa-1)} .
$$

Finally, if we choose a constant $C$ small enough and

$$
p=C(b-a)^{\kappa-1} / n^{\kappa^{2}}
$$

we have

$$
\Delta \leq \frac{b-a}{4 \cdot 3^{k}}
$$

and

$$
\omega^{*}(\mathscr{S}) \leq(1-p) \omega_{\mathrm{S}}^{*}+p\left(1-\frac{\alpha+(a+b) / 2}{2 \cdot 3^{k}}\right) .
$$

This concludes the proof of the theorem by choosing $\kappa$ large enough.

Finally, Theorem 1 follows by using the stabilizer for the four-qubit error detecting code in the game and noticing that the completeness and soundness has inverse polynomial gap in Theorem 23.

\section{Acknowledgments}

The author acknowledges helpful discussions with Richard Cleve, Debbie Leung, Fang Song, Thomas Vidick, Guoming Wang, John Watrous, Xiaodi Wu and Bei Zeng on related problems. This work is supported by NSERC and ARO. 


\section{References}

[1] A. Acín, N. Brunner, N. Gisin, S. Massar, S. Pironio, and V. Scarani, "Device-independent security of quantum cryptography against collective attacks”, Phys. Rev. Lett., vol. 98, p. 230 501, 23 2007. DOI: 10.1103/PhysRevLett.98.230501.

[2] D. Aharonov, I. Arad, and T. Vidick, The Quantum PCP Conjecture, arXiv:1309.7495, 2013.

[3] D. Aharonov, M. Ben-Or, and E. Eban, "Interactive proofs for quantum computations", in Innovations in Computer Science (ICS'10), 2010, pp. 453-469.

[4] D. Aharonov and T. Naveh, Quantum NP - A Survey, arXiv:quant-ph/0210077, 2002.

[5] S. Arora, C. Lund, R. Motwani, M. Sudan, and M. Szegedy, "Proof verification and the hardness of approximation problems”, J. ACM, vol. 45, no. 3, pp. 501-555, 1998. DOI: $10.1145 /$ 278298.278306.

[6] S. Arora and S. Safra, "Probabilistic Checking of Proofs: A New Characterization of NP", J. ACM, vol. 45, no. 1, pp. 70-122, 1998. DOI: 10.1145/273865. 273901.

[7] L Babai, “Trading group theory for randomness”, in Proceedings of the Seventeenth Annual ACM Symposium on Theory of Computing, ser. STOC '85, Providence, Rhode Island, USA: ACM, 1985, pp. 421-429. DOI: 10.1145/22145.22192.

[8] L. Babai, L. Fortnow, and C. Lund, "Nondeterministic exponential time has two-prover interactive protocols", in Proceedings of the 31st Annual Symposium on Foundations of Computer Science, ser. SFCS '90, Washington, DC, USA: IEEE Computer Society, 1990, pp. 1625. DOI: 10.1109/FSCS.1990.89520.

[9] J. Bell, “On the Einstein Podolsky Rosen Paradox", Physics, vol. 1, no. 3, pp. 195-200, 1964.

[10] M. Ben-Or, S. Goldwasser, J. Kilian, and A. Wigderson, "Multi-prover Interactive Proofs: How to Remove Intractability Assumptions", in Proceedings of the Twentieth Annual ACM Symposium on Theory of Computing, ser. STOC '88, Chicago, Illinois, USA: ACM, 1988, pp. 113-131. DOI: 10.1145/62212.62223.

[11] C. H. Bennett, D. P. DiVincenzo, J. A. Smolin, and W. K. Wootters, "Mixed-state entanglement and quantum error correction”, Phys. Rev. A, vol. 54, pp. 3824-3851, 5 1996. DOI: 10.1103/PhysRevA.54.3824.

[12] J. D. Biamonte and P. J. Love, "Realizable Hamiltonians for universal adiabatic quantum computers”, Phys. Rev. A, vol. 78, p. 012 352, 1 2008. DoI: 10.1103/PhysRevA.78. 012352.

[13] A. Broadbent, J. Fitzsimons, and E. Kashefi, "Universal blind quantum computation", in Foundations of Computer Science, 2009. FOCS '09. 50th Annual IEEE Symposium on, 2009, pp. 517-526. DOI: 10.1109/FOCS. 2009.36.

[14] P. J. Cameron, A. Montanaro, M. W. Newman, S. Severini, and A. Winter, "On the quantum chromatic number of a graph", Electronic Journal of Combinatorics, vol. 14, R81, 2007, See also arXiv:quant-ph/0608016.

[15] J. F. Clauser, M. A. Horne, A. Shimony, and R. A. Holt, "Proposed experiment to test local hidden-variable theories”, Phys. Rev. Lett., vol. 23, pp. 880-884, 15 1969. DOI: 10.1103 / PhysRevLett.23.880.

[16] R. Cleve, P. Hoyer, B. Toner, and J. Watrous, "Consequences and limits of nonlocal strategies", in Proceedings of the 19th Annual IEEE Conference on Computational Complexity, ser. CCC '04, Washington, DC, USA: IEEE Computer Society, 2004, pp. 236-249. DOI: 10 . 1109/CCC.2004.9. 
[17] R. Cleve and R. Mittal, "Characterization of binary constraint system games", in Automata, Languages, and Programming, J. Esparza, P. Fraigniaud, T. Husfeldt, and E. Koutsoupias, Eds., vol. 8572, ser. Lecture Notes in Computer Science, Springer Berlin Heidelberg, 2014, pp. 320-331. DOI: 10.1007/978-3-662-43948-7_27, See also arXiv:1209.2729.

[18] R. Colbeck, "Quantum and relativistic protocols for secure multi-party computation", $\mathrm{PhD}$ thesis, University of Cambridge, 2006, See also arXiv:0911.3814.

[19] S. A. Cook, "The complexity of theorem-proving procedures", in Proceedings of the third annual ACM symposium on Theory of computing, ser. STOC '71, Shaker Heights, Ohio, USA: ACM, 1971, pp. 151-158. DOI: 10.1145/800157.805047.

[20] T. S. Cubitt and A. Montanaro, "Complexity Classification of Local Hamiltonian Problems", in Proceedings of the 2014 IEEE 55th Annual Symposium on Foundations of Computer Science, ser. FOCS '14, Washington, DC, USA: IEEE Computer Society, 2014, pp. 120-129. DOI: 10.1109/FOCS . 2014.21.

[21] W. van Dam, F. Magniez, M. Mosca, and M. Santha, "Self-testing of universal and faulttolerant sets of quantum gates", in Proceedings of the Thirty-second Annual ACM Symposium on Theory of Computing, ser. STOC '00, Portland, Oregon, USA: ACM, 2000, pp. 688696. DOI: $10.1145 / 335305.335402$.

[22] I. Dinur, “The PCP Theorem by Gap Amplification”, J. ACM, vol. 54, no. 3, 2007. DOI: 10 . $1145 / 1236457.1236459$.

[23] J. Fitzsimons and T. Vidick, "A Multiprover Interactive Proof System for the Local Hamiltonian Problem", in Proceedings of the 2015 Conference on Innovations in Theoretical Computer Science, ser. ITCS '15, New York, NY, USA: ACM, 2015, pp. 103-112. DOI: 10 . 1145/ 2688073.2688094.

[24] J. F. Fitzsimons and E. Kashefi, Unconditionally verifiable blind computation, arXiv:1203.5217, 2012.

[25] S. Gharibian, Y. Huang, Z. Landau, and S. W. Shin, Quantum Hamiltonian Complexity, arXiv:1401.3916, 2014.

[26] S Goldwasser, S Micali, and C Rackoff, “The knowledge complexity of interactive proofsystems", in Proceedings of the Seventeenth Annual ACM Symposium on Theory of Computing, ser. STOC '85, Providence, Rhode Island, USA: ACM, 1985, pp. 291-304. DOI: 10 . $1145 / 22145.22178$.

[27] D. Gottesman, "Stabilizer Codes and Quantum Error Correction", PhD thesis, California Institute of Technology, 1997.

[28] T. Ito, H. Kobayashi, and K. Matsumoto, "Oracularization and two-prover one-round interactive proofs against nonlocal strategies", in Proceedings of the 2009 24th Annual IEEE Conference on Computational Complexity, ser. CCC '09, Washington, DC, USA: IEEE Computer Society, 2009, pp. 217-228. DOI: 10.1109/CCC . 2009. 22.

[29] T. Ito and T. Vidick, "A Multi-prover Interactive Proof for NEXP Sound Against Entangled Provers", in Proceedings of the 2012 IEEE 53rd Annual Symposium on Foundations of Computer Science, ser. FOCS '12, Washington, DC, USA: IEEE Computer Society, 2012, pp. 243252. DOI: 10.1109/FOCS . 2012.11.

[30] R. Jain, Z. Ji, S. Upadhyay, and J. Watrous, “QIP = PSPACE”, J. ACM, vol. 58, no. 6, p. 30, 2011. DOI: 10 . 1145/2049697 . 2049704, Preliminary version appeared in STOC '10. See also arXiv:0907.4737. 
[31] Z. Ji, Binary Constraint System Games and Locally Commutative Reductions, arXiv:1310.3794, 2013.

[32] C. Jordan, "Essai sur la géométrie à $n$ dimensions", Bulletin de la Société Mathématique de France, vol. 3, pp. 103-174, 1875.

[33] R. M. Karp, "Reducibility among combinatorial problems", in Complexity of Computer Computations, R. E. Miller and J. W. Thatcher, Eds., Plenum Press, New York, 1972, pp. 85103.

[34] J. Kempe, A. Kitaev, and O. Regev, "The Complexity of the Local Hamiltonian Problem", SIAM J. Comput., vol. 35, no. 5, pp. 1070-1097, 2006. DOI: 10.1137/S0097539704445226.

[35] J. Kempe, H. Kobayashi, K. Matsumoto, B. Toner, and T. Vidick, "Entangled games are hard to approximate", in Proceedings of the 2008 49th Annual IEEE Symposium on Foundations of Computer Science, ser. FOCS '08, Washington, DC, USA: IEEE Computer Society, 2008, pp. 447-456. DOI: 10.1109/FOCS. 2008.8.

[36] A. Y. Kitaev, Lecture given in Hebrew University, Jerusalem, Israel, 1999.

[37] A. Y. Kitaev, A. H. Shen, and M. N. Vyalyi, Classical and Quantum Computation. Boston, MA, USA: American Mathematical Society, 2002.

[38] A. Kitaev and J. Watrous, "Parallelization, amplification, and exponential time simulation of quantum interactive proof systems", in Proceedings of the Thirty-second Annual ACM Symposium on Theory of Computing, ser. STOC '00, Portland, Oregon, USA: ACM, 2000, pp. 608-617. DOI: 10.1145/335305.335387.

[39] H. Kobayashi and K. Matsumoto, "Quantum multi-prover interactive proof systems with limited prior entanglement”, Journal of Computer and System Sciences, vol. 66, no. 3, pp. 429450, 2003. DOI: $10.1016 / \mathrm{S} 0022-0000$ (03) 00035-7.

[40] S. B. Kochen and E. Specker, "The problem of hidden variables in quantum mechanics", Journal of Mathematics and Mechanics, vol. 17, pp. 59-87, 1967.

[41] R. Laflamme, C. Miquel, J. P. Paz, and W. H. Zurek, "Perfect quantum error correcting code”, Phys. Rev. Lett., vol. 77, pp. 198-201, 1 1996. DOI: 10.1103/PhysRevLett.77.198.

[42] Lance Fortnow and John Rompel and Michael Sipser, "On the power of multi-prover interactive protocols”, Theoretical Computer Science, vol. 134, no. 2, pp. 545-557, 1994. DOI: 10.1016/0304-3975(94)90251-8.

[43] L. Levin, "Universal search problems", Russian, Problems of Information Transmission, vol. 9, pp. 115-116, 31973.

[44] C. Lund, L. Fortnow, H. Karloff, and N. Nisan, "Algebraic methods for interactive proof systems”, J. ACM, vol. 39, no. 4, pp. 859-868, 1992. DOI: 10.1145/146585.146605.

[45] D. Mayers and A. Yao, "Quantum cryptography with imperfect apparatus", in Proceedings of the 39th Annual Symposium on Foundations of Computer Science, ser. FOCS '98, Washington, DC, USA: IEEE Computer Society, 1998, p. 503.

[46] M McKague, T. H. Yang, and V Scarani, "Robust self-testing of the singlet", Journal of Physics A: Mathematical and Theoretical, vol. 45, no. 45, p. 455304, 2012. DoI: 10 . 1088 / 1751 $8113 / 45 / 45 / 455304$. 
[47] M. McKague, “Self-testing graph states”, in Theory of Quantum Computation, Communication, and Cryptography, D. Bacon, M. Martin-Delgado, and M. Roetteler, Eds., vol. 6745, ser. Lecture Notes in Computer Science, Springer Berlin Heidelberg, 2014, pp. 104-120. DOI: $10.1007 / 978-3-642-54429-3 \_7$.

[48] N. D. Mermin, "Simple unified form for the major no-hidden-variables theorems", Physical Review Letters, vol. 65, pp. 3373-3376, 27 1990. DOI: 10.1103/PhysRevLett . 65. 3373.

[49] C. A. Miller and Y. Shi, Optimal robust quantum self-testing by binary nonlocal XOR games, arXiv:1207.1819, 2012.

[50] -, "Robust protocols for securely expanding randomness and distributing keys using untrusted quantum devices", in Proceedings of the 46th Annual ACM Symposium on Theory of Computing, ser. STOC '14, New York, New York: ACM, 2014, pp. 417-426. DOI: 10 .1145/ 2591796.2591843.

[51] R. Oliveira and B. M. Terhal, "The complexity of quantum spin systems on a two-dimensional square lattice", Quant. Inf. Comp., vol. 8, no. 10, pp. 0900-0924, 2008.

[52] T. J. Osborne, "Hamiltonian complexity", Reports on Progress in Physics, vol. 75, no. 2, p. $022001,2012$.

[53] A. Peres, "Incompatible results of quantum measurements", Physics Letters A, vol. 151, no. 3-4, pp. 107-108, 1990. DOI: 10.1016/0375-9601 (90)90172-K.

[54] S. Pironio, A. AcÃyn, S. Massar, A. B. de la Giroday, D. N. Matsukevich, P. Maunz, S. Olmschenk, D. Hayes, L. Luo, T. A. Manning, and C. Monroe, "Random numbers certified by Bell's theorem”, Nature, vol. 464, pp. 1021-1024, 7291 2010. DOI: 10.1038/nature09008.

[55] R. Raussendorf, D. E. Browne, and H. J. Briegel, "Measurement-based quantum computation on cluster states", Phys. Rev. A, vol. 68, p. 022 312, 2 2003. DOI: 10.1103 / PhysRevA . 68.022312.

[56] B. W. Reichardt, F. Unger, and U. Vazirani, “Classical command of quantum systems”, Nature, vol. 496, pp. 456-460, 7446 2013. DOI: 10 .1038/nature12035, See also arXiv:1209.0448.

[57] A. Shamir, "IP = PSPACE", J. ACM, vol. 39, no. 4, pp. 869-877, 1992. DOI: 10.1145/146585. 146609.

[58] Y. Shi, Both Toffoli and Controlled-NOT need little help to do universal quantum computation, arXiv:quant-ph/0205115, 2002.

[59] B. Tsirelson, "Quantum generalizations of Bell's inequality", Letters in Mathematical Physics, vol. 4, pp. 93-100, 1980.

[60] U. Vazirani and T. Vidick, "Certifiable Quantum Dice: Or, True Random Number Generation Secure Against Quantum Adversaries", in Proceedings of the Forty-fourth Annual ACM Symposium on Theory of Computing, ser. STOC '12, New York, New York, USA: ACM, 2012, pp. 61-76. DOI: 10.1145/2213977.2213984.

[61] —, "Fully device-independent quantum key distribution", Phys. Rev. Lett., vol. 113, p. 140 501, 14 2014. DOI: 10.1103/PhysRevLett.113.140501.

[62] T. Vidick, "Three-Player Entangled XOR Games Are NP-Hard to Approximate", 2013 IEEE 54th Annual Symposium on Foundations of Computer Science, pp. 766-775, 2013. DOI: 10 . 1109/FOCS . 2013.87. 
[63] J. Watrous, "PSPACE has constant-round quantum interactive proof systems", in Foundations of Computer Science, 1999. 40th Annual Symposium on, 1999, pp. 112-119. DoI: 10.1109/SFFCS. 1999.814583.

[64] R. F. Werner, "Quantum states with Einstein-Podolsky-Rosen correlations admitting a hiddenvariable model”, Phys. Rev. A, vol. 40, pp. 4277-4281, 8 1989. DoI: 10.1103/PhysRevA . 40. 4277. 